\title{
Improving the MILP-based Security Evaluation Algorithm against Differential/Linear Cryptanalysis Using A Divide-and-Conquer Approach
}

\author{
Chunning Zhou ${ }^{1,2}$, Wentao Zhang ${ }^{1,2}$, Tianyou Ding ${ }^{1,2}$ and Zejun Xiang ${ }^{3}$ \\ ${ }^{1}$ State Key Laboratory of Information Security, Institute of Information Engineering, Chinese \\ Academy of Sciences, Beijing, China, \{zhouchunning, zhangwentao, dingtianyou\}@iie.ac.cn \\ ${ }^{2}$ School of Cyber Security, University of Chinese Academy of Sciences, Beijing, China \\ ${ }^{3}$ Faculty of Mathematics and Statistics, Hubei Key Laboratory of Applied Mathematics, Hubei \\ University, Wuhan, China, xiangzejun@hubu.edu.cn
}

\begin{abstract}
In recent years, Mixed Integer Linear Programming (MILP) has been widely used in cryptanalysis of symmetric-key primitives. For differential and linear cryptanalysis, MILP can be used to solve two kinds of problems: calculation of the minimum number of differentially/linearly active S-boxes, and search for the best differential/linear characteristics. There are already numerous papers published in this area. However, the efficiency is not satisfactory enough for many symmetric-key primitives. In this paper, we greatly improve the efficiency of the MILP-based search algorithm for both problems. Each of the two problems for an $r$-round cipher can be converted to an MILP model whose feasible region is the set of all possible $r$ round differential/linear characteristics. Generally, high-probability differential/linear characteristics are likely to have a low number of active S-boxes at a certain round. Inspired by the idea of a divide-and-conquer approach, we divide the set of all possible differential/linear characteristics into several smaller subsets, then separately search them. That is to say, the search of the whole set is split into easier searches of smaller subsets, and optimal solutions within the smaller subsets are combined to give the optimal solution within the whole set. In addition, we use several techniques to further improve the efficiency of the search algorithm. As applications, we apply our search algorithm to five lightweight block ciphers: PRESENT, GIFT-64, RECTANGLE, LBLOCK and TWINE. For each cipher, we obtain better results than the best-known ones obtained from the MILP method. For the minimum number of differentially/linearly active S-boxes, we reach 31/31, 16/15, 16/16, 20/20 and 20/20 rounds for the five ciphers respectively. For the best differential/linear characteristics, we reach $18 / 18,15 / 13,15 / 14,16 / 15$ and $15 / 16$ rounds for the five ciphers respectively.
\end{abstract}

Keywords: Block cipher - Differential cryptanalysis - Linear cryptanalysis - MILP . Divide-and-conquer

\section{Introduction}

As a fundamental primitive of cryptography, block ciphers have received extensive attention from academia and industry. The most important criterion for designing a block cipher is to ensure that it can resist all known attacks, especially differential and linear cryptanalysis [BS91, Mat93]. 
To evaluate the security of a block cipher against differential/linear cryptanalysis, there are usually two approaches. One is to calculate the minimum number of differentially/linearly active S-boxes to obtain an upper bound of the maximum probability/absolute linear bias. The other approach is to search for the best differential/linear characteristics to calculate the maximum probability/absolute linear bias. For some block ciphers, e.g., for block ciphers with large block sizes, this needs a huge workload and is likely to be impossible to be accomplished in a reasonable time.

In [Mat94], Matsui proposed a branch and bound search algorithm to search for the best differential characteristic. Matsui's search algorithm is one of the most powerful and classic search tools, but difficult to implement in some cases. In recent years, a method based on Mixed Integer Linear Programming (MILP) is proposed to evaluate the security of recent symmetric-key primitive designs. Due to its easy-to-master and general-to-use features, the MILP-based method has been widely used.

In [WW11, MWGP11], for the first time, the authors applied MILP to evaluate the security of a block cipher against differential and linear cryptanalysis. Mouha et al. [MWGP11] introduced a model framework to calculate lower bounds of the minimum number of active S-boxes for word-oriented ciphers, then Sun et al. $\left[\mathrm{SHS}^{+} 13\right]$ extended their framework to SPN ciphers with bit-wise permutation diffusion layers. In [SHS $\left.{ }^{+} 13\right]$, bit-wise operations are described as linear inequalities. However, the description of an S-box is rough, which results in a solution of the model is not guaranteed to be a valid differential characteristic. At ASIACRYPT 2014, Sun et al. [SHW $\left.{ }^{+} 14 \mathrm{~b}\right]$ improved the MILP-based method for automatically evaluating the security of a block cipher against (related-key) differential cryptanalysis, and proposed a heuristic algorithm for finding actual (related-key) differential characteristics. They introduced two systematic methods for generating inequalities to describe the bit-wise S-box operation more accurately: logical condition modeling and convex hull computation. By using the two methods, some impossible differential characteristics are removed from the feasible region of the model, and tighter security bounds are obtained. Later in $\left[\mathrm{SHW}^{+} 14 \mathrm{a}\right]$, Sun et al. encoded differential probabilities/linear approximations of S-boxes into the MILP model, and argued that the feasible region of the model built by using the convex hull computation method for S-boxes is exactly the set of all possible (related-key) differential/linear characteristics. Therefore, their model can be used to obtain the minimum number of active S-boxes and find the best differential/linear characteristic. In $\left[\mathrm{FWG}^{+} 16\right], \mathrm{Fu}$ et al. extended the MILP-based automatic search algorithm to ARX ciphers and applied it to search for the best differential and linear characteristics for SPECK $\left[\mathrm{BSS}^{+} 13\right]$. Abdelkhalek et al. $\left[\mathrm{AST}^{+} 17\right]$ introduced a new method for modelling large S-boxes, e.g., 8-bit S-boxes, and evaluated the maximum probability of differential characteristics for SKINNY-128 [BJK $\left.{ }^{+} 16\right]$.

The problem of the calculation of the minimum number of differentially/linearly active S-boxes or the search for the best differential/linear characteristic for an $r$-round block cipher can be converted to an MILP model. As the size of the model increases significantly with the increasing of the number of rounds, the model can't be solved within a reasonable time when $r$ is too large. Thus a lot of papers are published to address this issue. A simple split approach was introduced in [MWGP11, SHW ${ }^{+} 14 \mathrm{~b}$ ], which splits $r$ rounds into the first $r_{1}$ and the last $\left(r-r_{1}\right)$ rounds then combines them, $1 \leq r_{1}<r$. In [SHQ $\left.{ }^{+} 15\right]$, the authors restricted difference patterns of S-boxes to search for improved differential characteristics. An interesting method that incorporates Matsui's branch and bound search algorithm and the MILP-based technique was introduced by Zhang et al. [ZSCH18]. They added the constraints derived from the bounding condition of Matsui's algorithm into the MILP model, which results in the model having a reduced feasible region and being solved with a shorter time. However, the efficiency of solving existing MILP models is still not satisfactory enough. New techniques are of necessity to push the limitation of our ability to evaluate the security of block ciphers based on MILP methods. Thus, the motivation of 
Improving the MILP-based Security Evaluation Algorithm against Differential/Linear 440 Cryptanalysis Using A Divide-and-Conquer Approach

this paper is to make the MILP-based method an efficient enough tool upon which we can entirely rely to evaluate the security of block ciphers against differential/linear attack.

Our Contributions. Due to the duality between differential and linear cryptanalysis [Mat94], we mainly focus on the security evaluation algorithm against differential cryptanalysis. Calculating the minimum number of active S-boxes or searching for the best differential characteristic is equivalent to an MILP-based search for the optimal differential characteristic with the minimum number of active S-boxes or with the minimum weight (defined as the negative of the binary logarithm of the probability). Our main contributions are:

1. We propose an improved MILP-based search algorithm to evaluate the security of block ciphers against differential cryptanalysis. Inspired by the idea of a divideand-conquer approach, we divide the set of all possible differential characteristics into smaller subsets, then separately search them. We observe that high-probability differential characteristics are likely to have a low number of active S-boxes at a certain round, thus smaller subsets are partitioned based on the information of S-boxes. Take an SPN cipher as an example, we assume that the differential characteristics in a subset have exactly one or two active S-boxes at a certain round. By using the MILP technique, searching a subset can be transformed into an MILP model whose feasible region is exactly the subset. Then the optimal differential characteristics within the subsets are combined to give the optimal differential characteristic within the whole set. To further improve efficiency, we use three techniques:

(a) At the beginning of the search, we generate a valid differential characteristic and use it as the currently optimal differential characteristic. Its number of active S-boxes (or weight) is served as an upper bound of the minimum number of active S-boxes (or weight). The currently optimal differential characteristic and the upper bound are dynamically updated during the search.

(b) When searching a subset, we calculate a lower bound of the minimum number of active S-boxes (or weight) within it. If the lower bound is greater than or equal to the upper bound introduced in the first technique, there is no better differential characteristic and we terminate the search of this subset;

(c) We choose a proper search order of subsets, such that better differential characteristics can be searched as early as possible.

Finally, an MILP-based search algorithm is proposed. The algorithm can be used to both calculate the minimum number of active S-boxes and search for the best differential characteristic. Also, the algorithm can be extended to the security evaluation of block ciphers against linear cryptanalysis with a slight modification.

2. We apply our search algorithm to five lightweight block ciphers: PRESENT [BKL $\left.{ }^{+} 07\right]$, GIFT-64 [BPP ${ }^{+}$17], RECTANGLE [ZBL ${ }^{+}$15], LBLOCK [WZ11] and TWINE [SMMK12]. For each cipher, we obtain better results than previous best-known results obtained from the MILP method. For the minimum number of differentially/linearly active S-boxes, we reach $31 / 31,16 / 15,16 / 16,20 / 20$ and 20/20 rounds for the five ciphers respectively. For the best differential/linear characteristics, we reach 18/18, 15/13, 15/14, 16/15 and 15/16 rounds for the five ciphers respectively. To compare with previous MILP-based work, we implement Sun et al.'s model [SHW $\left.{ }^{+} 14 \mathrm{~b}, \mathrm{SHW}^{+} 14 \mathrm{a}\right]$ and Zhang et al.'s model [ZSCH18] to search for the best differential characteristic for the five ciphers. We implement the three methods (ours, Sun et al.'s and Zhang et al.'s) on a PC, and summarize the experimental results in Table 1. From the table, we see that our search algorithm has an advantage over the other two methods when the number of rounds is large, and for each cipher, our 
Table 1: Comparison of the results on the best differential and linear characteristics

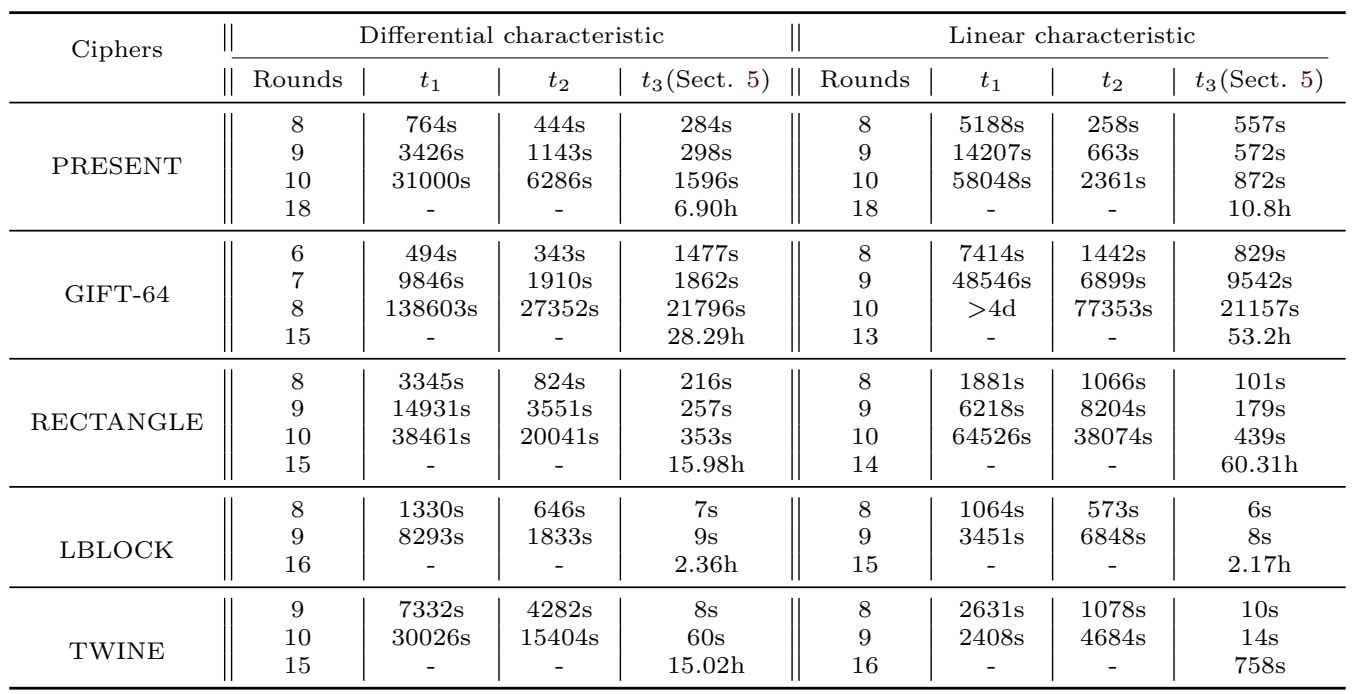

$t_{1}$ and $t_{2}$ respectively denote the time of solving Sun et al.'s model $\left.\left[\mathrm{SHW}^{+} 14 \mathrm{~b}, \mathrm{SHW}^{+} 14 \mathrm{a}\right]\right)$ and Zhang et al. 's model [ZSCH18], and $t_{3}$ denotes the runtime of our search algorithm. For the three methods, we use Sun's Greedy algorithm $\left.\left[\mathrm{SHW}^{+}{ }^{14 a}\right]\right)$ to reduce the number of inequalities from the convex-hull modeling, and use Gurobi software [Gur] with MipFocus equal to 2 to solve models.

search algorithm covers more rounds with less time. The source code is available at https://github.com/Chunning-Zhou/MILPBasedSearchAlgorithmDiff.

Organization. In Section 2, we recall the existing automatic MILP-based tool for evaluating the security of block ciphers against differential cryptanalysis. An improved MILP-based search algorithm by incorporating the idea of a divide-and-conquer approach is proposed in Section 3. We use our search algorithm to evaluate the security of block ciphers against differential/linear cryptanalysis, and apply it to five lightweight block ciphers in Sections 4 and 5. In Section 6, we conclude the paper and provide some ideas for future work. More details and experimental results are given in Appendices.

\section{Related Work}

Calculating the minimum number of active S-boxes and searching for the best differential characteristic are two ways to evaluate the security of block ciphers against differential attacks. In this section, we introduce the MILP-based method for solving the two problems.

\subsection{Model Framework for Calculating the Minimum Number of Active S-boxes}

Mouha et al. [MWGP11] introduced a model framework to calculate lower bounds of the minimum number of active S-boxes for word-oriented block ciphers. Then, Sun et al. extended their model framework to bit-oriented block ciphers $\left[\mathrm{SHS}^{+} 13, \mathrm{SHW}^{+} 14 \mathrm{~b}\right.$, $\left.\mathrm{SHW}^{+} 14 \mathrm{a}\right]$.

\subsubsection{Mouha et al.'s Model Framework for Word-Oriented Block Ciphers}

Mouha et al. [MWGP11] considered truncated differences, and used a 0-1 variable to describe a word-level difference, such that the variable equals 1 if and only if the input 
word is non-zero. Assume that a cipher is composed of three operations: XOR, linear transformation and S-box, the following constraints are introduced to describe word-level difference propagations through a cipher.

Equations Describing the XOR Operation. Let $a, b$ and $c$ denote word-level input and corresponding output differences of the XOR operation, the following equations are used to describe the XOR operation:

$$
\left\{\begin{array}{l}
a+b+c \geq 2 d_{\oplus} \\
d_{\oplus} \geq a, d_{\oplus} \geq b, d_{\oplus} \geq c
\end{array}\right.
$$

where $d_{\oplus}$ is a dummy variable taking values in $\{0,1\}$.

Equations Describing the Linear Transformation. Let $\left(x_{0}, \ldots, x_{m-1}\right)$ and $\left(y_{0}, \ldots, y_{m-1}\right)$ denote word-level input and output differences of the linear transformation $L$ respectively. Given the differential branch number $\mathcal{B}_{\mathcal{D}}$ of $L$, the linear transformation is described by:

$$
\left\{\begin{array}{l}
\sum_{k=0}^{m-1} x_{k}+\sum_{k=0}^{m-1} y_{k} \geq \mathcal{B}_{\mathcal{D}} d_{L}, \\
d_{L} \geq x_{k}, d_{L} \geq y_{k}, k \in\{0, \ldots, m-1\}
\end{array}\right.
$$

where $d_{L}$ is a dummy variable taking values in $\{0,1\}$. In $\left[\mathrm{SHS}^{+} 13\right]$, the authors pointed out that if $L$ is not MDS, additional constraints are needed to ensure that non-zero input difference must result in non-zero output difference and vice versa. The constraints are similar to Equation (4) describing an S-box.

Additional Constraints. To avoid a trivial solution, it needs an additional constraint to ensure that at least one S-box is active. Besides, all dummy $d$-variables, and the variables representing the plaintext differences are restricted to be $0-1$ variables.

Objective Function. The objective function is to minimize the number of active S-boxes, i.e, the sum of all variables representing word-level input differences of S-boxes of each round.

By using the model framework above, an MILP model is built to calculate a lower bound of the minimum number of active S-boxes for a word-oriented block cipher. However, this framework did not consider bit-wise operations, thus it is not applicable to bit-oriented block ciphers.

\subsubsection{Sun et al.'s Bit-wise Model Framework}

In $\left[\mathrm{SHS}^{+} 13, \mathrm{SHW}^{+} 14 \mathrm{~b}, \mathrm{SHW}^{+} 14 \mathrm{a}\right]$, Sun et al. described operations of bit-oriented block ciphers. They used a 0-1 variable to denote the bit-level difference, such that the variable equals 1 if and only if the bit-level difference is non-zero. For each S-box, they used a 0-1 variable $A$ to denote the word-level input difference, such that $A=1$ if and only if the input word of the S-box is non-zero. Therefore, the objective function is to minimize the sum of all $A$ variables.

For bit-wise XOR, an additional inequality $a+b+c \leq 2$ is added into Equation (1). For an $w \times v$ S-box marked by $A$, suppose $\left(x_{0}, \ldots, x_{w-1}\right)$ and $\left(y_{0}, \ldots, y_{v-1}\right)$ are input and output differences respectively, the following constrains are introduced to describe the S-box. Firstly, $A=1$ holds if and only if $x_{0}, \ldots, x_{w-1}$ are not all zero:

$$
\left\{\begin{array}{l}
A-x_{k} \geq 0, k \in\{0, \ldots, w-1\} \\
x_{0}+x_{1}+\ldots+x_{w-1}-A \geq 0
\end{array}\right.
$$


Besides, the Hamming weight of $\left(x_{0}, \ldots, x_{w-1}, y_{0}, \ldots, y_{v-1}\right)$ is greater than or equal to the branch number $\mathcal{B}_{\mathcal{S}}$ of the $\mathrm{S}$-box for a non-zero input difference:

$$
\left\{\begin{array}{l}
\sum_{k=0}^{w-1} x_{k}+\sum_{k=0}^{v-1} y_{k} \geq \mathcal{B}_{\mathcal{S}} d_{S}, \\
d_{S} \geq x_{k}, k \in\{0, \ldots, w-1\} \\
d_{S} \geq y_{k}, k \in\{0, \ldots, v-1\}
\end{array}\right.
$$

where $d_{S}$ is a dummy variable taking values in $\{0,1\}$, and the branch number $\mathcal{B}_{\mathcal{S}}$ of an S-box is defined as

$$
\mathcal{B}_{\mathcal{S}}=\min _{a \neq b}\left\{\operatorname{wt}((a \oplus b) \| S(a) \oplus S(b)): a, b \in \mathbb{F}_{2}^{w}\right\}
$$

where $\operatorname{wt}(\cdot)$ is the standard Hamming weight. In addition, for bijective S-boxes, non-zero input difference must result in non-zero output difference and vice versa:

$$
\left\{\begin{array}{l}
w y_{0}+w y_{1}+\ldots+w y_{v-1}-\left(x_{0}+x_{1}+\ldots x_{w-1}\right) \geq 0 \\
v x_{0}+v x_{1}+\ldots+v x_{w-1}-\left(y_{0}+y_{1}+\ldots y_{v-1}\right) \geq 0
\end{array}\right.
$$

By using the objective function and the constraints introduced above, an MILP model is built to calculate a lower bound of the minimum number of active S-boxes for a bit-oriented cipher. The constraints used to describe S-boxes are rough, thus a feasible solution of the model is not guaranteed to be a valid differential characteristic.

To describe an S-box more accurately, Sun et al. $\left[\mathrm{SHW}^{+} 14 \mathrm{~b}\right]$ proposed two systematic methods for generating inequalities: the logical condition modeling and the convex hull computation. The inequalities are used to remove invalid differential characteristics from the feasible region of the model. Later in $\left[\mathrm{SHW}^{+} 14 \mathrm{a}\right]$, Sun et al. proved that the feasible region of the model built by using the convex hull computation method for S-boxes is exactly the set of all possible differential characteristics. In the convex hull computation method, a possible difference propagation $\left(x_{0}, \ldots, x_{w-1}\right) \rightarrow\left(y_{0}, \ldots, y_{v-1}\right)$ of an $w \times v$ S-box is treated as a point in $\mathbb{F}_{2}^{w+v}:\left(x_{0}, \ldots, x_{w-1}, y_{0}, \ldots, y_{v-1}\right) \in \mathbb{F}_{2}^{w+v}$. All possible difference propagations of the S-box constitute a set of finitely many discrete points. By computing the H-Representation of the convex hull of the set with the help of SageMath software [sag], inequalities are generated and their feasible solutions are exactly the points in the set. The number of inequalities computed from SageMath is generally very large, thus a greedy algorithm $\left[\mathrm{SHW}^{+}{ }^{14 a}\right.$ ] and an MILP-based reduction algorithm [ST17] were proposed to select a small number of inequalities. By using these reduced inequalities to describe the S-box, all impossible difference propagations of S-boxes are removed from the feasible region of the model. By modeling S-boxes with the convex hull computation method and restricting all variables involved to be $0-1$ variables, the MILP model can be used to obtain the minimum number of active S-boxes.

\subsection{Model Framework for Searching for the Best Differential Charac- teristic}

In $\left[\mathrm{SHW}^{+}{ }^{14 \mathrm{a}}\right]$, Sun et al. encoded differential probabilities of an S-box into an MILP model and searched for the best differential characteristic for a block cipher.

Take PRESENT cipher as an example. PRESENT uses a $4 \times 4$ S-box, and there are 3 nontrivial probabilities in the difference distribution table of the S-box. A possible difference propagation of an S-box $\left(x_{0}, x_{1}, x_{2}, x_{3}\right) \rightarrow\left(y_{0}, y_{1}, y_{2}, y_{3}\right)$ with the probability $\operatorname{Pr}$ is treated as a 10-dimensional point:

$$
\left(x_{0}, x_{1}, x_{2}, x_{3}, y_{0}, y_{1}, y_{2}, y_{3}, p_{0}, p_{1}\right) \in \mathbb{F}_{2}^{10}
$$


where

$$
\left(p_{0}, p_{1}\right)=\left\{\begin{array}{l}
(0,0), \text { if } \operatorname{Pr}=1 ; \\
(0,1), \text { if } \operatorname{Pr}=2^{-2} \\
(1,1), \text { if } \operatorname{Pr}=2^{-3}
\end{array}\right.
$$

that is to say, $\operatorname{Pr}=2^{-\left(p_{0}+2 p_{1}\right)}$. By using the convex hull computation method, inequalities are generated to describe all the possible points. To find the best differential characteristic, i.e., the differential characteristic with the maximal probability, the objective function is to minimize the sum of all $\left(p_{0}+2 p_{1}\right)$. Note that in Equation (5), $p_{1}$ equals 1 if and only if the input difference is non-zero, thus $p_{1}$ also indicates the input word of the S-box.

\section{Improving the MILP-based Search Algorithm by Incor- porating with A Divide-and-Conquer Approach}

The difficulty of the problem of the calculation of the minimum number of active S-boxes or the search for the best differential characteristic usually increases exponentially with the increasing of the number of rounds. Existing MILP models can hardly be used to solve the problem when the number of rounds is large. The main reason is that in existing MILP models built for an $r$-round cipher, the feasible region is the set of all possible $r$-round differential characteristics. Inspired by the idea of a divide-and-conquer approach, we improve the efficiency of the MILP-based search algorithm basing on our empirical knowledge about the valid differential characteristics. We first divide the set of all possible $r$-round differential characteristics into several subsets, we then search each subset separately. By using the MILP technique, searching a subset can be equivalent to solving an MILP model whose feasible region is exactly the subset. Then the optimal differential characteristic within the whole set is given by combining all the results returned from subsets. To further improve efficiency, we introduce several techniques. Finally, an improved MILP-based search algorithm is proposed. For the convenience of illustration, we mainly focus on searching for the best differential characteristic for SPN ciphers. The search for Feistel ciphers is similar and it is illustrated in Appendix A.

\subsection{Dividing the Set of All Possible Differential Characteristics}

Unlike Feistel ciphers, for SPN ciphers, valid differential characteristics have at least one active S-box in each round, and those with the highest probability are likely to have at most two active S-boxes at a certain round. Based on this observation, we propose a proper way to divide the set of all possible differential characteristics into smaller subsets then conquer them separately.

For an $r$-round SPN cipher, we first divide the set of all possible $r$-round differential characteristics into three kinds of subsets:

Subset-1 In this kind of subsets, differential characteristics have at least one active S-box in each round, and there is at least one round that contains exactly one active S-box;

Subset-2 In this kind of subsets, differential characteristics have at least two active Sboxes in each round, and there is at least one round that contains exactly two active S-boxes;

Subset-3 In this subset, differential characteristics have at least three active S-boxes in each round.

For Subset-1 and Subset-2, we further divide them by fixing the index $i(i=1,2, \ldots, r)$, such that round $i$ has exactly 1 or 2 active S-boxes. By doing this, the number of all 
possible input differences of round $i$ decreases significantly. Then, we traverse the input difference of round $i$ to further divide the subsets.

For the sake of brevity, we use $\mathcal{D}_{r, N_{A}, i, \Delta}$ to denote the subset of $r$-round differential characteristics that satisfy the following two constraints:

Constraint 1 there are at least $N_{A}$ active S-boxes in each round except for round $i$;

Constraint 2 the input difference of round $i$ equals $\Delta$.

Specially, when $i=0$, there is no constraint on the input difference, and we ignore the second constraint by (not rigorously) writing $\Delta=0$. Differential characteristics in the subset are only required to satisfy the Constraint 1, i.e., there are at least $N_{A}$ active S-boxes in each round. Moreover, if $i=r+1$, we regard the constraint of "input difference of round $(r+1)$ equals $\Delta$ " as the constraint of "output difference of round $r$ equals $\Delta$ ".

In summary, the set of all possible $r$-round differential characteristics for an $r$-round SPN cipher is divided into the following subsets:

$$
\left(\bigcup_{N_{A}, i, \Delta} \mathcal{D}_{r, N_{A}, i, \Delta}\right) \bigcup \mathcal{D}_{r, 3,0,0}
$$

where $N_{A} \in\{1,2\}, i \in\{1,2, \ldots, r\}, \Delta$ belongs to the set of all possible input differences of round $i$ that has exactly $N_{A}$ active S-boxes. Take PRESENT as an example, its block size is 64 bits and it uses a $4 \times 4 \mathrm{~S}$-box. Thus it applied $16 \mathrm{~S}$-boxes in parallel in each round. When one round has exactly one active S-box, there are $16 \times\left(2^{4}-1\right)=240$ possibilities of input differences of this round; when one round has exactly two active S-boxes, there are $120 \times\left(2^{4}-1\right)^{2}=27000$ possibilities of the input difference of this round. Based on Equation (6), the set of all possible differential characteristics for $r$-round PRESENT is divided into $(r \times 240+r \times 27000+1)$ subsets.

\subsection{Building MILP Models for Searching Subsets}

By using the MILP technique described in Section 2, searching for the best differential characteristic for an $r$-round cipher is transformed into an MILP model. The feasible region of this model is the set of all possible $r$-round differential characteristics. To search for the best differential characteristic within a subset divided in Equation (6), we introduce additional constraints and build an MILP model whose feasible region is exactly the subset.

For the subset named $\mathcal{D}_{r, N_{A}, i, \Delta}$, differential characteristics in it are constrained by Constraints 1 and 2. Let $A_{j, k}$ denote the word-level input difference of the $k$ th S-box at round $j$, the Constraint 1 is described by Equation (7):

$$
\sum_{k=0}^{N_{S}-1} A_{j, k} \geq N_{A}, j \in\{1,2, . ., r\}, j \neq i,
$$

where $N_{S}$ is the total number of S-boxes in each round. Moreover, let $x_{i, j}$ denote the $j$ th bit input difference of round $i$, the Constraint 2 is described by Equation (8):

$$
x_{i, j}=\Delta_{j}, j \in\{0,1, \ldots, n-1\},
$$

where $\Delta_{j}$ is the $j$ th bit of $\Delta$, and $n$ is the block size of the cipher. By adding Equations $(7,8)$ into the original MILP model that is used to search for the best $r$-round differential characteristic, we obtain a model whose feasible region is the subset $\mathcal{D}_{r, N_{A}, i, \Delta}$. By solving this model, we obtain the best differential characteristic within the subset.

By separately searching all the subsets divided, namely, solving all the corresponding MILP models, the best differential characteristics within the subsets are returned. They 
Improving the MILP-based Security Evaluation Algorithm against Differential/Linear 446

Cryptanalysis Using A Divide-and-Conquer Approach

are combined to give the best differential characteristic for an $r$-round cipher. Although the model with a smaller feasible region is easier to be solved, it will cost a huge amount of time if we solve all the models. This is mainly because the number of the subsets divided is generally large and some of the models are time-consuming (e.g., the model whose feasible region is $\mathcal{D}_{r, 3,0,0}$ takes a long time to be solved). Therefore, we introduce several techniques to further improve the efficiency of the algorithm in the next section.

\subsection{Techniques to further Improve Efficiency}

In this section, we introduce some techniques to improve the efficiency of our search algorithm. With the help of the techniques, the search of a subset can be early terminated, thus the runtime of the algorithm is greatly reduced.

The weight of a differential characteristic is defined as the negative of the binary logarithm of the probability. Searching for the best differential characteristic for an $r$ round cipher is equivalent to searching for the $r$-round differential characteristic with the minimum weight. For the search for $r$ rounds, three techniques are used:

Technique 1. Setting an Upper Bound. Similar to the strategy used in [Mat94, $\mathrm{AST}^{+} 17$, at the beginning of the search, we generate a valid $r$-round differential characteristic. This differential characteristic is used as the currently best $r$-round differential characteristic, and its weight is served as an upper bound of the minimum weight. We generate such an $r$-round differential characteristic by exploiting one of the best $(r-1)$ round differential characteristics we have found. The difference pattern of an S-box is defined as its truncated difference, i.e., a 0-1 variable. In the model built for searching for the best $r$-round differential characteristic, we fix the S-boxes of the first $(r-1)$ rounds (or the last $(r-1)$ rounds) to have the same difference patterns as those of the best $(r-1)$-round differential characteristic. By solving this model, we obtain an $r$-round differential characteristic and its weight. To reduce unnecessary searches, we only focus on the subsets in which the differential characteristics have weights smaller than the obtained upper bound. During the search of subsets, if we find an $r$-round differential characteristic having a weight smaller than the current upper bound, the currently best $r$-round differential characteristic is updated by it and the current upper bound is updated by its weight.

Technique 2. Calculating Lower Bounds within Subsets. The upper bound introduced in Technique 1 uses the weight of the currently best differential characteristic as a threshold. When searching a subset, we calculate a lower bound of the minimum weight of the differential characteristics within it. If the lower bound is greater than or equal to the upper bound, there is no differential characteristic with a weight smaller than the weight of the currently best differential characteristic. At this point, we terminate the search of this subset and search the next one. With the help of this technique, the number of MILP models to be solved is reduced.

Technique 3. Choosing a Proper Search Order of Subsets. In order to make Technique 2 more efficient, we aim to find better differential characteristics as early as possible. Thus, we choose a proper search order of the subsets divided in Equation (6). There are two cases we can take into account: (1) we preferentially search the subsets which are more likely to provide better differential characteristics; (2) we preferentially search the subsets whose corresponding MILP models can be solved with less time. According to the two cases, we traverse the subsets $\mathcal{D}_{r, N_{A}, i, \Delta}$ in a proper order, where $N_{A} \in\{1,2\}, i \in\{1,2, \ldots, r\}, \Delta$ belongs to the set of all possible input differences of round $i$ that has exactly $N_{A}$ active S-boxes. By default, $N_{A}$ is traversed from 1 to 2 (i.e., $N_{A} \in[1,2]$ ), namely, we first search 
the subsets $\mathcal{D}_{r, 1, i, \Delta}$ then $\mathcal{D}_{r, 2, i, \Delta}$. If we can predict that the best differential characteristics have at least two active S-boxes in each round from some prior knowledge, we preferentially search the subsets $\mathcal{D}_{r, 2, i, \Delta}$ (i.e., $N_{A} \in[2,1]$ ). The search complexity usually increases exponentially with the increasing of the number of rounds. By our empirical observation, the closer the $i$ is to the middle of $r$, the smaller the complexity of searching the subset $\mathcal{D}_{r, N_{A}, i, \Delta}$. This might due to the input difference of round $i$ is fixed to equal $\Delta$, which results in the complexity of searching the subset is determined by the sum of complexities of searching the first $(i-1)$ rounds and the last $(r-i+1)$ rounds. Therefore, we traverse the index $i$ by:

$$
i \in \operatorname{SearchR}=\left\{\begin{array}{l}
{\left[\frac{r+2}{2}, \frac{r+2}{2}-1, \frac{r+2}{2}+1, \ldots, 1\right], \text { if } r \text { is even; }} \\
\left\lfloor\left\lfloor\frac{r+2}{2}\right\rfloor,\left\lfloor\frac{r+2}{2}\right\rfloor+1,\left\lfloor\frac{r+2}{2}\right\rfloor-1, \ldots, 1\right], \text { if } r \text { is odd } .
\end{array}\right.
$$

For example, when $r=10, \operatorname{SearchR}=[6,5,7,4,8,3,9,2,10,1]$; and when $r=11, i \in$ SearchR $=[6,7,5,8,4,9,3,10,2,11,1]$.

\subsection{Methods for Calculating Lower Bounds within Subsets}

Based on Technique 2 introduced in Section 3.3, the search of a subset is terminated if a lower bound of the minimum weight of the differential characteristics within the subset is bigger than or equal to the weight of the currently best differential characteristic. In this section, we provide several methods to calculate the lower bound.

For an $r$-round SPN cipher, the set of all possible $r$-round differential characteristics is divided into several subsets according to Equation (6). For the subset named $\mathcal{D}_{r, N_{A}, i, \Delta}$, we use

$$
\mathrm{LB}\left[r, N_{A}, i, \Delta\right]
$$

to store a lower bound of the minimum weight of the differential characteristics within it, where $N_{A} \in\{1,2,3\}, i \in\{0,1, \ldots, r+1\}, \Delta \in \mathbb{F}_{2}^{n}$ ( $n$ is the block size of the cipher). The array LB is called the lower bound array in which the values are dynamically and repeatedly updated. In the following, we provide three methods for estimating lower bounds of the minimum weight of the differential characteristics within $\mathcal{D}_{r, N_{A}, i, \Delta}$. Methods 1-2 use other values that have been determined and stored in the lower bound array LB, and Method 3 uses the results returned from MILP models. The three methods can be used to assign a value to $\operatorname{LB}\left[r, N_{A}, i, \Delta\right]$, and they are written as Functions AssignByMethod1(), AssignByMethod2() and AssignByMethod3() respectively.

Method 1. By using the simple split method introduced in [MWGP11, $\mathrm{SHW}^{+} 14 \mathrm{~b}$ ], we split $r$ rounds into the first $r_{1}$ rounds and the last $\left(r-r_{1}\right)$ rounds then combine the two smaller parts, $1 \leq r_{1}<r$.

1. For the subset $\mathcal{D}_{r, N_{A}, 0,0}$, the differential characteristics of the first $r_{1}$ rounds and the last $\left(r-r_{1}\right)$ rounds respectively belong to $\mathcal{D}_{r_{1}, N_{A}, 0,0}$ and $\mathcal{D}_{r-r_{1}, N_{A}, 0,0}$. Thus the lower bound within $\mathcal{D}_{r, N_{A}, 0,0}$ can be estimated by:

$$
\max _{1 \leq r_{1} \leq r-1}\left(\mathrm{LB}\left[r_{1}, N_{A}, 0,0\right]+\mathrm{LB}\left[r-r_{1}, N_{A}, 0,0\right]\right) .
$$

2. For the subset $\mathcal{D}_{r, N_{A}, i, \Delta}$ with $i \in\{1, \ldots, r\}, \Delta \neq 0$, the input difference of round $i$, namely, the output difference of round $(i-1)$ equals $\Delta$. Thus the lower bound within $\mathcal{D}_{r, N_{A}, i, \Delta}$ can be estimated by:

$$
\max \left\{\begin{array}{l}
\mathrm{LB}\left[i-1, N_{A}, i, \Delta\right]+\mathrm{LB}\left[r-i+1, N_{A}, 1, \Delta\right],(i \geq 2), \\
\max _{1 \leq r_{1} \leq i-1}\left(\mathrm{LB}\left[r_{1}, N_{A}, 0,0\right]+\mathrm{LB}\left[r-r_{1}, N_{A}, i-r_{1}, \Delta\right]\right),(i \geq 2), \\
\max _{i \leq r_{1} \leq r-1}\left(\mathrm{LB}\left[r_{1}, N_{A}, i, \Delta\right]+\mathrm{LB}\left[r-r_{1}, N_{A}, 0,0\right]\right)
\end{array}\right\} .
$$


Improving the MILP-based Security Evaluation Algorithm against Differential/Linear 448 Cryptanalysis Using A Divide-and-Conquer Approach

3. For the subset $\mathcal{D}_{r, N_{A}, r+1, \Delta}$, the lower bound within it can be estimated by:

$$
\max _{1 \leq r_{1} \leq r-1}\left(\mathrm{LB}\left[r_{1}, N_{A}, r_{1}+1, \Delta\right]+\mathrm{LB}\left[r-r_{1}, N_{A}, 0,0\right]\right)
$$

Method 2. When searching the subset $\mathcal{D}_{r, N_{A}, i, \Delta}, N_{A} \in\{1,2\}, i \in \operatorname{SearchR}, \Delta$ belongs to the set of all possible input differences of round $i$ that has exactly $N_{A}$ active S-boxes, we avoid searching the differential characteristics that have been searched. Take the search for $r=10$ as an example, SearchR $=[6,5,7,4,8,3,9,2,10,1]$. Firstly, we search the subsets $\mathcal{D}_{r, N_{A}, 6, \Delta}$. After searching $\mathcal{D}_{r, N_{A}, 6, \Delta}, \forall \Delta$, we begin to deal with the subsets $\mathcal{D}_{r, N_{A}, 5, \Delta}$. At this point, the differential characteristics satisfying round 6 with exactly $N_{A}$ active S-boxes have already been searched. To avoid duplicate searches, we assume that the differential characteristics to be searched later have at least $\left(N_{A}+1\right)$ active S-boxes at round 6 . We add the additional constraint into the differential characteristics in $\mathcal{D}_{r, N_{A}, 5, \Delta}$, and we calculate a lower bound of the minimum weight of these differential characteristics. If the lower bound is greater than or equal to the weight of the currently best $r$-round differential characteristic, there is no better differential characteristic than the currently best one within $\mathcal{D}_{r, N_{A}, 5, \Delta}$. Suppose $i=\operatorname{SearchR}[j], 1 \leq j \leq r-1$, let $R_{\text {front }}$ denote the number in front of $i$, i.e., $R_{\text {front }}=\operatorname{SearchR}[j-1]$. In the following, we provide a method to estimate a lower bound of the minimum weight of the differential characteristics satisfying the additional constraint.

- For the case $i<\operatorname{SearchR}[0]$, the differential characteristics in $\mathcal{D}_{r, N_{A}, i, \Delta}$ satisfy the additional constraint: "there are at least $\left(N_{A}+1\right)$ active S-boxes in each of rounds from $(i+1)$ to $R_{\text {front }}$ ". Similar to Method 1 , we split $r$ rounds into smaller parts then combine them. Thus a lower bound of the minimum weight of the differential characteristics with the additional constraint is estimated by:

$$
\max \left\{\begin{array}{l}
\left.\mathrm{LB}\left[i, N_{A}, 0,0\right]+\mathrm{LB}\left[R_{\text {front }}-i, N_{A}+1,0,0\right]+\mathrm{LB}\left[r-R_{\text {front }}, N_{A}, 0,0\right]\right), \\
\left.\mathrm{LB}\left[i-1, N_{A}, i, \Delta\right]+\mathrm{LB}\left[R_{\text {front }}-i+1, N_{A}+1,1, \Delta\right]+\mathrm{LB}\left[r-R_{\text {front }}, N_{A}, 0,0\right]\right)
\end{array}\right\},
$$

where in the first expression, the $r$ rounds are split into three parts: (1) round 1 to $i$; (2) round $(i+1)$ to $R_{\text {front }}$; $(3)$ round $\left(R_{\text {front }}+1\right)$ to $r$; and in the second expression, the $r$ rounds are split into: (1) round 1 to $(i-1)$; (2) round $i$ to $R_{\text {front }}$; (3) round $\left(R_{\text {front }}+1\right)$ to $r$.

- For the case $i>\operatorname{SearchR}[0]$, the differential characteristics in $\mathcal{D}_{r, N_{A}, i, \Delta}$ satisfy the additional constraint: "there are at least $\left(N_{A}+1\right)$ active S-boxes in each of rounds from $R_{\text {front }}$ to $(i-1)$ ". Similar to the first case, we estimate a lower bound of the minimum weight of the differential characteristics with the additional constraint by:

$$
\max \left\{\begin{array}{l}
\left.\mathrm{LB}\left[R_{\text {front }}-1, N_{A}, 0,0\right]+\mathrm{LB}\left[i-R_{\text {front }}, N_{A}+1,0,0\right]+\mathrm{LB}\left[r-i+1, N_{A}, 0,0\right]\right), \\
\left.\mathrm{LB}\left[R_{\text {front }}-1, N_{A}, 0,0\right]+\mathrm{LB}\left[i-R_{\text {front }}, N_{A}+1, i-R_{\text {front }}+1, \Delta\right]+\mathrm{LB}\left[r-i+1, N_{A}, 1, \Delta\right]\right)
\end{array}\right\} .
$$

Moreover, tighter lower bounds can be estimated by splitting the $r$ rounds more carefully, and the details and implementation can be seen in the public source code.

Method 3. We estimate lower bounds of the minimum weight of the differential characteristics within a subset by solving MILP models. The constraints for describing a subset have been introduced in Section 3.2, and there are two kinds of MILP models for us to choose from:

1. The first kind of model is called a "Rough" model that provides a rough lower bound. We notice that the models built by using Mouha et al.'s [MWGP11] and Sun et 
al.'s $\left[\mathrm{SHS}^{+}\right.$13] model frameworks are solved quickly, and they are used to calculate a lower bound of the minimum number of active S-boxes for word-oriented and bit-oriented ciphers respectively. By solving this model, a rough lower bound of the minimum weight is obtained by multiplying the number of active S-boxes with the minimum weight of a single S-box. Note that if the cipher is bit-oriented and its branch number of an S-box equal to 2, the constraints for modelling an S-box is insufficient, and this model can't provide a useful lower bound.

2. The second kind of model is called a "Tightest" model that provides the tightest lower bound (i.e., the lower bound is exactly the minimum weight). The model built by using Sun et al.'s model framework $\left[\mathrm{SHW}^{+} 14 \mathrm{~b}, \mathrm{SHW}^{+} 14 \mathrm{a}\right]$ is used to search for the best differential characteristic, and it returns the minimum weight.

\subsection{Improved Search Algorithm}

Based on the approaches and techniques presented in Section 3.1 to Section 3.4, we are ready to present an MILP-based search algorithm for searching for the best differential characteristics for a block cipher, as illustrated in Algorithm 1. In the following, we give a detailed description of the algorithm.

Given a block cipher and a number of rounds $R$, Algorithm 1 is used to search for the best $r$-round differential characteristic, $r \in\{1,2, \ldots, R\}$. At the beginning of the search for $r$ rounds, we generate the currently best $r$-round differential characteristic and obtain an upper bound of the minimum weight (denoted as UpperBound) according to Technique 1. Nextly, we initialize the lower bound array LB and search the subsets divided for the $r$-round cipher. During the search, we dynamically update the currently best $r$-round differential characteristic and UpperBound. After searching all the subsets, the currently best $r$-round differential characteristic is exactly the best $r$-round differential characteristic, and UpperBound equals the minimum weight for the $r$-round cipher.

Function InitLBArray(). This function is used to initialize the lower bound array LB, specifically, assign values to $\operatorname{LB}\left[r, N_{A}, 0,0\right], N_{A}=1,2,3$. These values will be used later when searching the subsets divided in Equation (6). To assign values to $\operatorname{LB}\left[r, N_{A}, 0\right.$, 0$]$, we estimate lower bounds of the minimum weight of the differential characteristics within $\mathcal{D}_{r, N_{A}, 0,0}$ by using Methods 1 and 3 (introduced in Section 3.4). In Method 3, a "Rough" model or a "Tightest" model is solved. The "Rough" model is usually easier to be solved than the "Tightest" model for the same subset, thus we preferentially solve the "Rough" model to obtain a lower bound. If the lower bound returned from the "Rough" model is a good one, we don't need to solve the "Tightest" model. Usually, either of the two models will be time-consuming when the number of rounds $r$ exceeds a certain value. Thus Method 3 is adopted only when $r$ is small which makes it possible to solve the model in a short time.

Function SearchSubset12(). This function is used to search the subsets $\mathcal{D}_{r, N_{A}, i, \Delta}$, where $N_{A} \in\{1,2\}, i \in$ SearchR, $\Delta$ belongs to the set of all possible input differences of round $i$ that has exactly $N_{A}$ active S-boxes. We traverse the parameters $N_{A}$ and $i$ according to Technique 3 , and search a specific subset $\mathcal{D}_{r, N_{A}, i, \Delta}$ in Line 30-36. According to Technique 2 , we calculate a lower bound of the minimum weight of the differential characteristics within the subset and use it to determine whether there is a differential characteristic better than the currently best one. We first estimate the lower bound within $\mathcal{D}_{r, N_{A}, i, \Delta}$ by using the value stored in $\mathrm{LB}\left[r, N_{A}, 0,0\right]$ based on the fact that all differential characteristics in $\mathcal{D}_{r, N_{A}, i, \Delta}$ belong to $\mathcal{D}_{r, N_{A}, 0,0}$, and we store it in $\operatorname{LB}\left[r, N_{A}, i, \Delta\right]$. Then, the value stored in $\mathrm{LB}\left[r, N_{A}, i, \Delta\right]$ is updated by using Method 1 and Method 2. There is no model solved until 


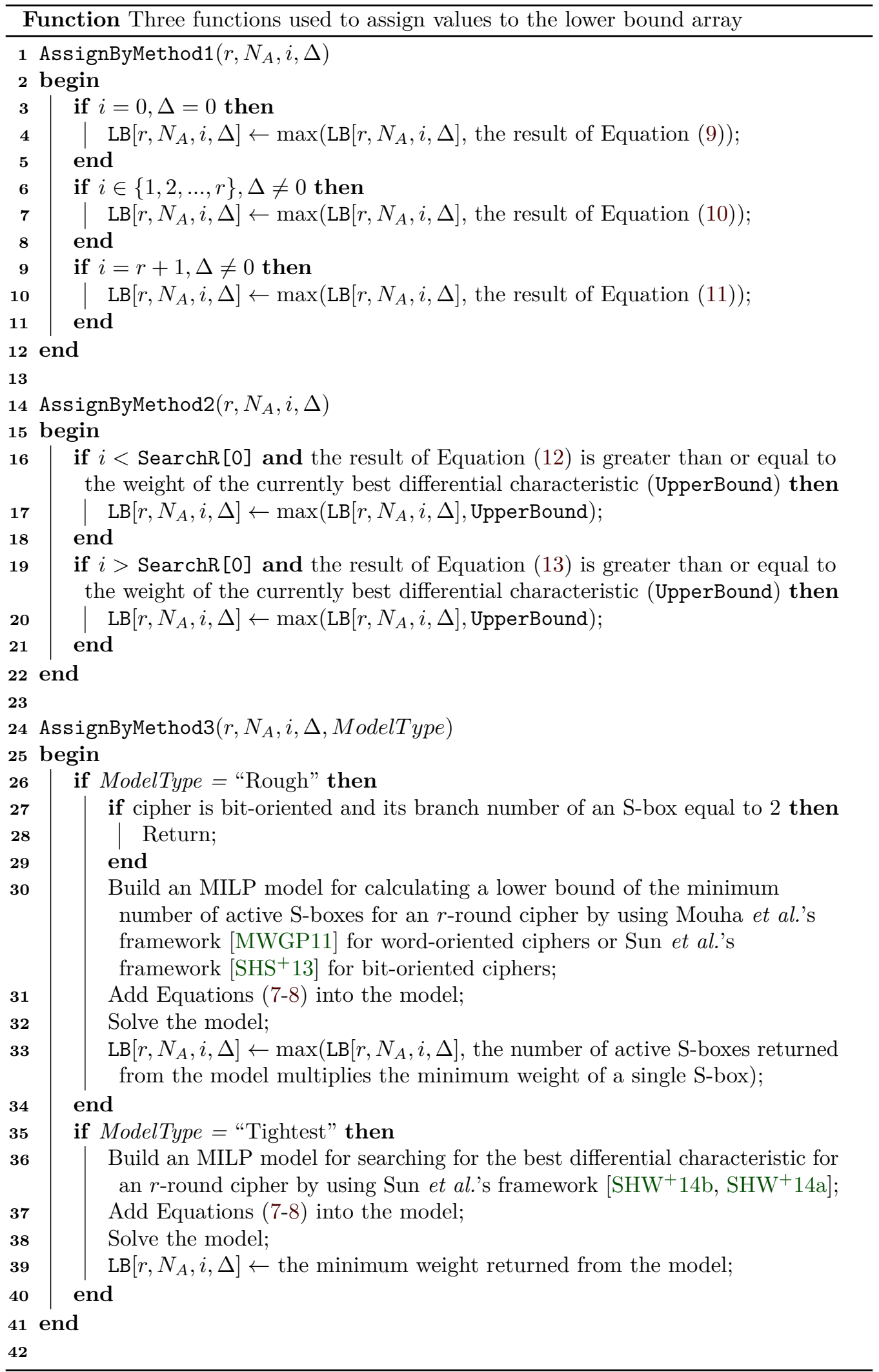


now. If the current value stored in $\operatorname{LB}\left[r, N_{A}, i, \Delta\right]$ is greater than or equal to the weight of the currently best $r$-round differential characteristic (UpperBound), we terminate the search of this subset and search the next one. Otherwise, we estimate a tighter lower bound by calling Function UpdateLBSubset12(), and update the value stored in $\operatorname{LB}\left[r, N_{A}, i, \Delta\right]$.

Function UpdateLBSubset12(). In this function, we estimate lower bounds of the minimum weight of the differential characteristics within the subset $\mathcal{D}_{r, N_{A}, i, \Delta}$ by solving MILP models, where $N_{A} \in\{1,2\}, i \in$ SearchR, $\Delta$ belongs to the set of all possible input differences of round $i$ that has exactly $N_{A}$ active S-boxes. We observe that the running time of solving an MILP model usually increases dramatically as the number of rounds increases. Therefore, we first solve a model built for the subset of $r_{1}$-round differential characteristics, then use Method 1 to estimate lower bounds within the subsets of $r_{2}$-round differential characteristics, $r_{1}$ increases from $1, r_{2}=r_{1}+1, \ldots, R$. For each $r_{1}$, we obtain a new lower bound within the subset $\mathcal{D}_{r, N_{A}, i, \Delta}$. The lower bound is constantly updated by increasing the number $r_{1}$, until it is greater than or equal to UpperBound or it equals the minimum weight of the differential characteristics within the subset. After calling this function, if the value stored in $\operatorname{LB}\left[r, N_{A}, i, \Delta\right]$ is still smaller than UpperBound, we find a differential characteristic that has a weight smaller than the weight of the currently best $r$-round differential characteristic.

Function SearchSubset3(). This function is used to search the subset $\mathcal{D}_{r, 3,0,0}$. A lower bound of the minimum weight of the differential characteristics within $\mathcal{D}_{r, 3,0,0}$ had been calculated and it was stored in $\operatorname{LB}[r, 3,0,0]$. For most lightweight SPN ciphers, the best differential characteristics are likely to have exactly 1 or 2 active S-boxes at a certain round. The value stored in $\operatorname{LB}[r, 3,0,0]$ is generally greater than or equal to UpperBound. In this case, the search of the subset is terminated and no other model needs to be solved.

\section{Automatic Security Evaluation against Differential/Linear Cryptanalysis}

In this section, we use our improved search algorithm to evaluate the security of block ciphers against differential and linear Cryptanalysis.

\subsection{Security Evaluation Against Differential Cryptanalysis}

Algorithm 1 is used to search for the best differential characteristics for a block cipher. It can be used to calculate the minimum number of active S-boxes by modifying the aim of searching for the differential characteristic with the minimum weight to that with the minimum number of active S-boxes. At this point, the lower bound array LB is used to store a lower bound of the minimum number of active S-boxes. Besides, the "Tightest" model in Method 3 is built to calculate the minimum number of active S-boxes by using Sun et al.'s framework $\left[\mathrm{SHW}^{+} 14 \mathrm{~b}, \mathrm{SHW}^{+} 14 \mathrm{a}\right]$. Applying our algorithm to an $r$-round block cipher, we obtain the minimum weight or its lower bound calculated from the minimum number of active S-boxes. If the minimum weight or its lower bound is greater than or equal to cipher's block size, it can be concluded that the $r$-round cipher is secure against differential cryptanalysis.

\subsection{Security Evaluation Against Linear Cryptanalysis}

According to the duality between differential and linear cryptanalysis [Mat94], Algorithm 1 is easily extended to the security evaluation against linear cryptanalysis. The linear 


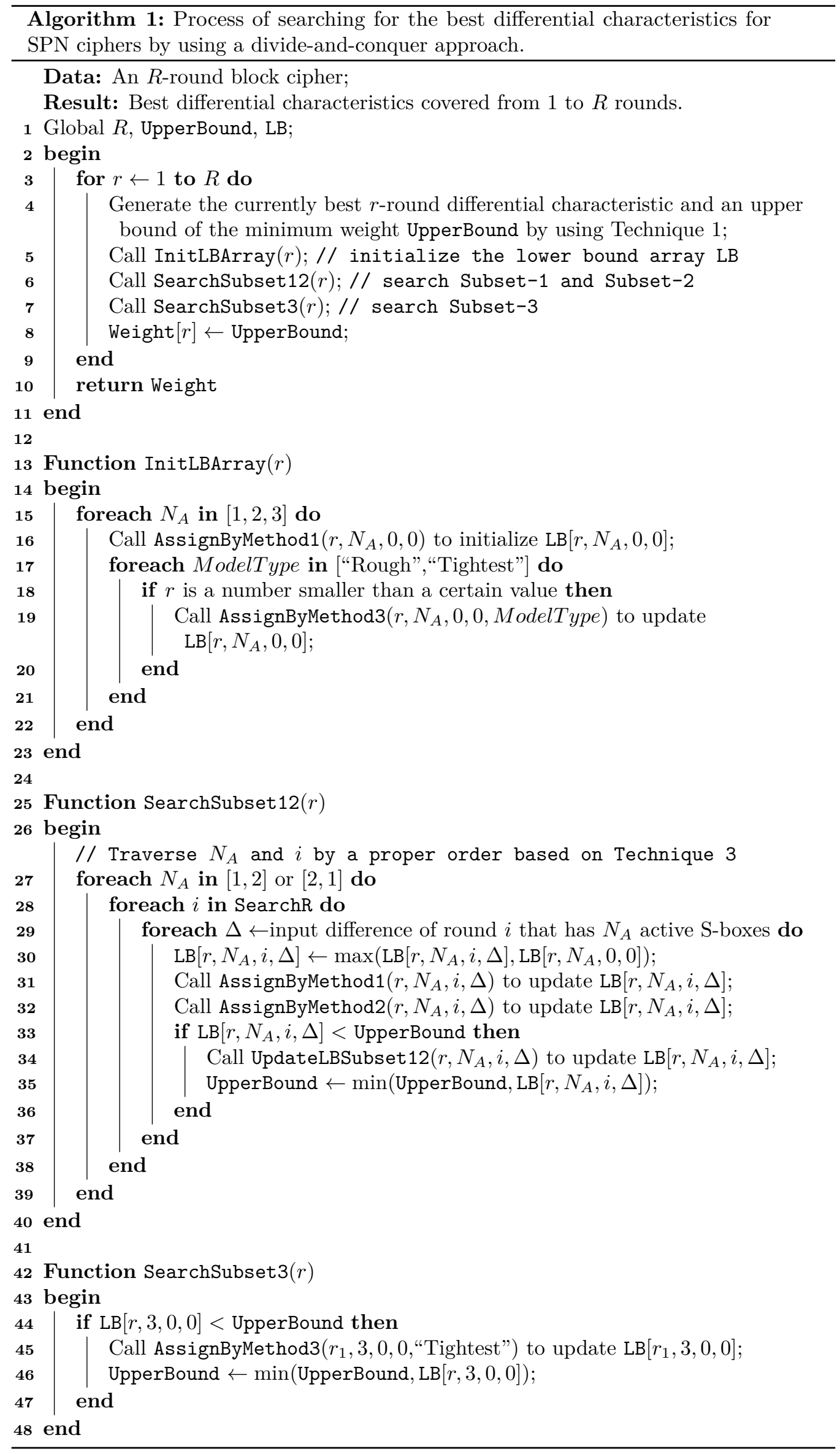




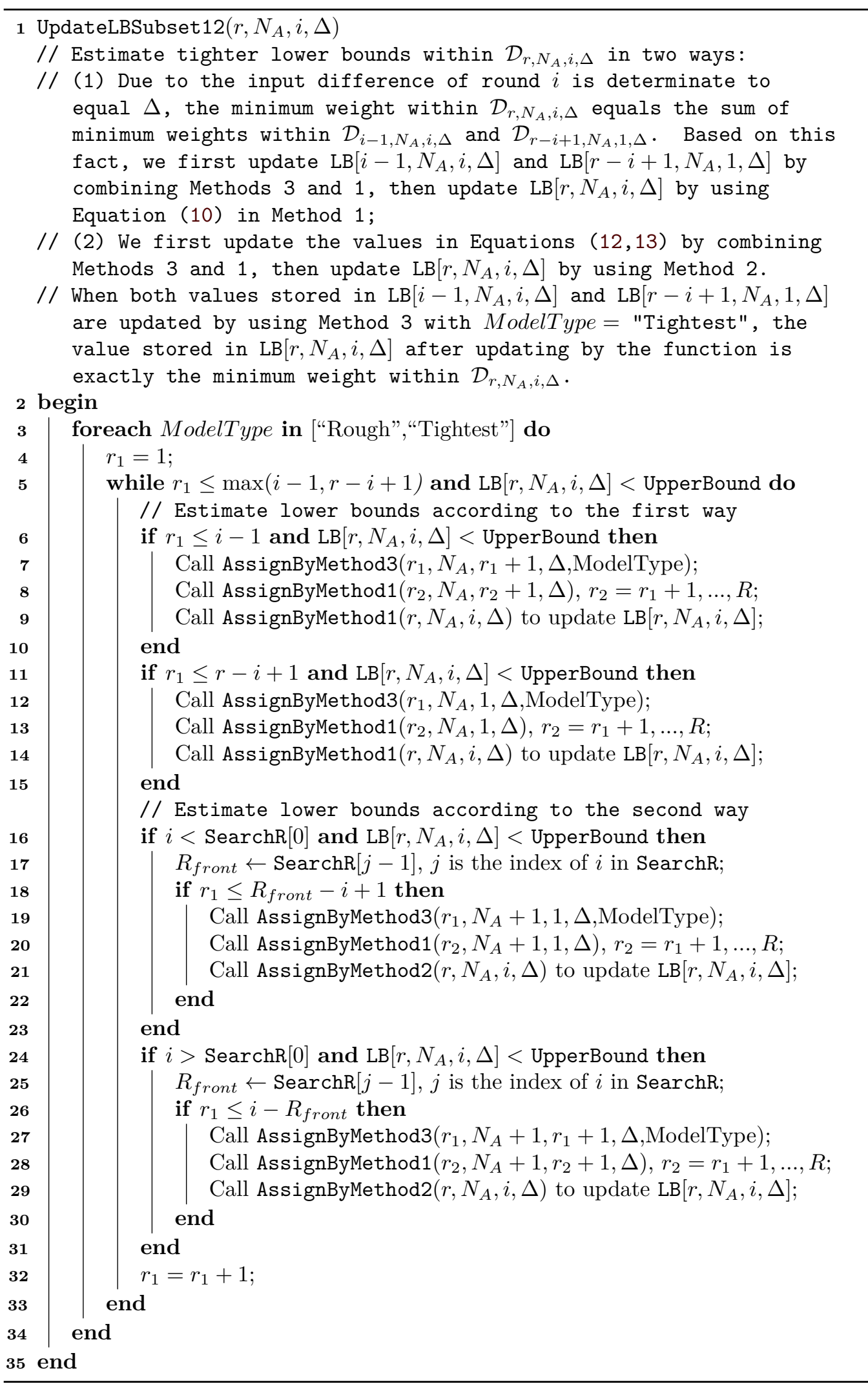


Improving the MILP-based Security Evaluation Algorithm against Differential/Linear 454

Cryptanalysis Using A Divide-and-Conquer Approach

characteristics with the maximum absolute linear bias are likely to have a low number of active S-boxes at a certain round, thus we divide the set of all possible linear characteristics into smaller subsets by using the partition method introduced for differential characteristics. In [MWGP11, $\left.\mathrm{SHS}^{+} 13, \mathrm{SHW}^{+} 14 \mathrm{~b}, \mathrm{SHW}^{+} 14 \mathrm{a}\right]$, the authors described linear mask propagations through a block cipher, and built MILP models to search for the best linear characteristic and calculate the minimum number of linearly active S-boxes. By using their work, we build an MILP model to search each of the subsets divided. For linear characteristics, the weight is defined as the negative of the binary logarithm of the correlation contribution. Searching for the best linear characteristic or calculating the minimum number of active S-boxes for an $r$-round cipher is transformed into the search for the $r$-round linear characteristic with the minimum weight or with the minimum number of active S-boxes. If the minimum weight or its lower bound calculated from the minimum number of active S-boxes is greater than or equal to the half of cipher's block size, it can be concluded that the $r$-round cipher is secure against linear cryptanalysis.

\section{Applications to PRESENT, GIFT-64, RECTANGLE, LBLOCK and TWINE}

In this section, we apply our search algorithm to five lightweight block ciphers: three SPN ciphers PRESENT, GIFT-64, RECTANGLE and two Feistel ciphers LBLOCK, TWINE. For each of the five ciphers, we obtain the minimum number of differentially and linearly active S-boxes and find the best differential and linear characteristics. The experimental results are summarized in Tables 2-6, where \#\{AS$D\}$ and $\#\left\{A S_{L}\right\}$ respectively denote the minimum number of differentially and linearly active S-boxes, $\operatorname{Pr}_{D}$ denotes the probability of the best differential characteristic, and $\operatorname{Cor}_{L}$ denotes the correlation contribution of the best linear characteristic. We study the number of MILP models to be solved in Appendix $\mathrm{C}$, and give examples of best differential and linear characteristics in Appendix D.

Our experiment is performed on a PC (Intel(R) Core(TM) i7-4790 CPU, 3.60 GHz, 10.00GB RAM, 4 cores, Linux), and we use the openly available software Gurobi [Gur] to solve MILP models. We observe that in the experiment, the running time is usually improved when setting MipFocus (a parameter in Gurobi) to 2. Therefore, we implement our algorithm with setting MipFocus equal to 2, and we obtain the results in Table 1 by using the same parameter for a fair comparison. We recommend readers to refer [Gur] for more information and select appropriate parameters for other problems.

\subsection{PRESENT}

PRESENT is an SPN cipher designed by Bogdanov et al. [BKL $\left.{ }^{+} 07\right]$. The differential and linear branch numbers of PRESENT S-box are equal to 3 and 2 respectively. For PRESENT, we obtain the minimum number of differentially/linearly active S-boxes for up to $31 / 31$ rounds (full rounds) and find the best differential/linear characteristics for up to $18 / 18$ rounds.

Although our results on the minimum number of differentially active S-boxes are the same as those in $\left[\mathrm{SHS}^{+} 13\right]$, they didn't prove the results are exact values because their description for modelling an S-box is rough, while ours is exact. In [Wan08], the authors provided the best differential characteristics for PRESENT for 5 to 10 rounds, and good ones for 11 to 15 rounds, while we cover more rounds. Although the weight of the best differential characteristic for 15-round PRESENT is larger than the block size 64, it can be used to analyze the differential clustering [DR02] of PRESENT, whose clustering effect is very strong as shown in [WSTP12]. 
Table 2: Experimental results of PRESENT

\begin{tabular}{|c|c|c|c|c|c|c|c|c|c|}
\hline \multirow{2}{*}{ Rounds } & \multicolumn{4}{|c|}{ Differential cryptanalysis } & $\|$ & \multicolumn{4}{|c|}{ Linear cryptanalysis } \\
\hline & $\#\left\{A S_{D}\right\}$ & Time & $\operatorname{Pr}_{D}$ & Time & $\|$ & $\#\left\{A S_{L}\right\}$ & Time & $\operatorname{Cor}_{L}$ & Time \\
\hline 1 & 1 & $0 \mathrm{~s}$ & $2^{-2}$ & $1 \mathrm{~s}$ & & 1 & $0 \mathrm{~s}$ & $2^{-1}$ & $0 \mathrm{~s}$ \\
\hline 2 & 2 & $1 \mathrm{~s}$ & $2^{-4}$ & $2 \mathrm{~s}$ & & 2 & $2 \mathrm{~s}$ & $2^{-2}$ & $2 \mathrm{~s}$ \\
\hline 3 & 4 & $2 \mathrm{~s}$ & $2^{-8}$ & $3 \mathrm{~s}$ & & 3 & $3 \mathrm{~s}$ & $2^{-4}$ & $71 \mathrm{~s}$ \\
\hline 4 & 6 & $4 \mathrm{~s}$ & $2^{-12}$ & $4 \mathrm{~s}$ & & 4 & $6 \mathrm{~s}$ & $2^{-6}$ & $88 \mathrm{~s}$ \\
\hline 5 & 10 & $5 \mathrm{~s}$ & $2^{-20}$ & $5 \mathrm{~s}$ & & 5 & $9 \mathrm{~s}$ & $2^{-8}$ & $152 \mathrm{~s}$ \\
\hline 6 & 12 & $8 \mathrm{~s}$ & $2^{-24}$ & $249 \mathrm{~s}$ & & 6 & $8 \mathrm{~s}$ & $2^{-10}$ & $128 \mathrm{~s}$ \\
\hline 7 & 14 & $10 \mathrm{~s}$ & $2^{-28}$ & $9 \mathrm{~s}$ & & 7 & $7 \mathrm{~s}$ & $2^{-12}$ & $18 \mathrm{~s}$ \\
\hline 8 & 16 & $11 \mathrm{~s}$ & $2^{-32}$ & $11 \mathrm{~s}$ & & 8 & $8 \mathrm{~s}$ & $2^{-14}$ & $98 \mathrm{~s}$ \\
\hline 9 & 18 & $15 \mathrm{~s}$ & $2^{-36}$ & $14 \mathrm{~s}$ & & 9 & $10 \mathrm{~s}$ & $2^{-16}$ & $15 \mathrm{~s}$ \\
\hline 10 & 20 & $16 \mathrm{~s}$ & $2^{-41}$ & $1298 \mathrm{~s}$ & & 10 & $11 \mathrm{~s}$ & $2^{-18}$ & $300 \mathrm{~s}$ \\
\hline 11 & 22 & $18 \mathrm{~s}$ & $2^{-46}$ & $438 \mathrm{~s}$ & & 11 & $12 \mathrm{~s}$ & $2^{-20}$ & $11 \mathrm{~s}$ \\
\hline 12 & 24 & $22 \mathrm{~s}$ & $2^{-52}$ & $311 \mathrm{~s}$ & & 12 & $14 \mathrm{~s}$ & $2^{-22}$ & $978 \mathrm{~s}$ \\
\hline 13 & 26 & $24 \mathrm{~s}$ & $2^{-56}$ & $22 \mathrm{~s}$ & & 13 & $15 \mathrm{~s}$ & $2^{-24}$ & $14 \mathrm{~s}$ \\
\hline 14 & 28 & $31 \mathrm{~s}$ & $2^{-62}$ & $18859 \mathrm{~s}$ & & 14 & $17 \mathrm{~s}$ & $2^{-26}$ & $3507 \mathrm{~s}$ \\
\hline 15 & 30 & $32 \mathrm{~s}$ & $2^{-66}$ & $2594 \mathrm{~s}$ & & 15 & $19 \mathrm{~s}$ & $2^{-28}$ & $16 \mathrm{~s}$ \\
\hline 16 & 32 & $19 \mathrm{~s}$ & $2^{-70}$ & $370 \mathrm{~s}$ & & 16 & $21 \mathrm{~s}$ & $2^{-30}$ & $3080 \mathrm{~s}$ \\
\hline 17 & 34 & $20 \mathrm{~s}$ & $2^{-74}$ & $20 \mathrm{~s}$ & & 17 & $23 \mathrm{~s}$ & $2^{-32}$ & $16302 \mathrm{~s}$ \\
\hline 18 & 36 & $22 \mathrm{~s}$ & $2^{-78}$ & $629 \mathrm{~s}$ & & 18 & $24 \mathrm{~s}$ & $2^{-34}$ & $14105 \mathrm{~s}$ \\
\hline 19 & 38 & $34 \mathrm{~s}$ & & & & 19 & $26 \mathrm{~s}$ & & \\
\hline 20 & 40 & $29 \mathrm{~s}$ & & & & 20 & $28 \mathrm{~s}$ & & \\
\hline 21 & 42 & $28 \mathrm{~s}$ & & & & 21 & $30 \mathrm{~s}$ & & \\
\hline 22 & 44 & $29 \mathrm{~s}$ & & & & 22 & $34 \mathrm{~s}$ & & \\
\hline 23 & 46 & $37 \mathrm{~s}$ & & & & 23 & $35 \mathrm{~s}$ & & \\
\hline 24 & 48 & $34 \mathrm{~s}$ & & & & 24 & $37 \mathrm{~s}$ & & \\
\hline 25 & 50 & $36 \mathrm{~s}$ & & & & 25 & $40 \mathrm{~s}$ & & \\
\hline 26 & 52 & $38 \mathrm{~s}$ & & & & 26 & $42 \mathrm{~s}$ & & \\
\hline 27 & 54 & $40 \mathrm{~s}$ & & & & 27 & $44 \mathrm{~s}$ & & \\
\hline 28 & 56 & $42 \mathrm{~s}$ & & & & 28 & $46 \mathrm{~s}$ & & \\
\hline 29 & 58 & $42 \mathrm{~s}$ & & & & 29 & $49 \mathrm{~s}$ & & \\
\hline 30 & 60 & $44 \mathrm{~s}$ & & & & 30 & $49 \mathrm{~s}$ & & \\
\hline 31 & 62 & $47 \mathrm{~s}$ & & & & 31 & $51 \mathrm{~s}$ & & \\
\hline Total time & \multicolumn{2}{|c|}{$740 \mathrm{~s}$} & \multicolumn{2}{|c|}{$6.90 \mathrm{~h}$} & & \multicolumn{2}{|c|}{$720 \mathrm{~s}$} & \multicolumn{2}{|c|}{$10.8 \mathrm{~h}$} \\
\hline
\end{tabular}

Table 3: Experimental results of GIFT-64

\begin{tabular}{|c|c|c|c|c|c|c|c|c|}
\hline \multirow[t]{2}{*}{ Rounds } & \multicolumn{4}{|c|}{ Differential cryptanalysis } & \multicolumn{4}{|c|}{ Linear cryptanalysis } \\
\hline & $\| \#\left\{A S_{D}\right\}$ & Time & $\operatorname{Pr}_{D}$ & Time & $\| \#\left\{A S_{L}\right\}$ & Time & $\| \operatorname{Cor}_{L}$ & Time \\
\hline 1 & 1 & $1 \mathrm{~s}$ & $2^{-1.415}$ & $1 \mathrm{~s}$ & 1 & $0 \mathrm{~s}$ & $2^{-1}$ & $0 \mathrm{~s}$ \\
\hline 2 & 2 & $2 \mathrm{~s}$ & $2^{-3.415}$ & $47 \mathrm{~s}$ & 2 & $1 \mathrm{~s}$ & $2^{-2}$ & $2 \mathrm{~s}$ \\
\hline 3 & 3 & $3 \mathrm{~s}$ & $2^{-7}$ & $108 \mathrm{~s}$ & 3 & $3 \mathrm{~s}$ & $2^{-3}$ & $3 \mathrm{~s}$ \\
\hline 4 & 5 & $69 \mathrm{~s}$ & $2^{-11.415}$ & $291 \mathrm{~s}$ & 5 & $61 \mathrm{~s}$ & $2^{-5}$ & $77 \mathrm{~s}$ \\
\hline 5 & 7 & $61 \mathrm{~s}$ & $2^{-17}$ & $849 \mathrm{~s}$ & 7 & $60 \mathrm{~s}$ & $2^{-7}$ & $99 \mathrm{~s}$ \\
\hline 6 & 10 & $144 \mathrm{~s}$ & $2^{-22.415}$ & $181 \mathrm{~s}$ & 9 & $65 \mathrm{~s}$ & $2^{-10}$ & $160 \mathrm{~s}$ \\
\hline 7 & 13 & $115 \mathrm{~s}$ & $2^{-28.415}$ & $385 \mathrm{~s}$ & 12 & $177 \mathrm{~s}$ & $2^{-13}$ & $225 \mathrm{~s}$ \\
\hline 8 & 16 & $271 \mathrm{~s}$ & $2^{-38}$ & $19934 \mathrm{~s}$ & 15 & $243 \mathrm{~s}$ & $2^{-16}$ & $263 \mathrm{~s}$ \\
\hline 9 & 18 & $28 \mathrm{~s}$ & $2^{-42}$ & $32 \mathrm{~s}$ & 18 & $493 \mathrm{~s}$ & $2^{-20}$ & $8713 \mathrm{~s}$ \\
\hline 10 & 20 & $124 \mathrm{~s}$ & $2^{-48}$ & $7569 \mathrm{~s}$ & 20 & $681 \mathrm{~s}$ & $2^{-25}$ & $11615 \mathrm{~s}$ \\
\hline 11 & 22 & $77 \mathrm{~s}$ & $2^{-52}$ & $121 \mathrm{~s}$ & 22 & $392 \mathrm{~s}$ & $2^{-29}$ & $34019 \mathrm{~s}$ \\
\hline 12 & 24 & $19 \mathrm{~s}$ & $2^{-58}$ & $61001 \mathrm{~s}$ & 24 & $3206 \mathrm{~s}$ & $2^{-31}$ & $14644 \mathrm{~s}$ \\
\hline 13 & 26 & $75 \mathrm{~s}$ & $2^{-62}$ & $604 \mathrm{~s}$ & 26 & $11229 \mathrm{~s}$ & $2^{-34}$ & $121716 \mathrm{~s}$ \\
\hline 14 & 28 & $15 \mathrm{~s}$ & $2^{-68}$ & $9121 \mathrm{~s}$ & 28 & $7982 \mathrm{~s}$ & & \\
\hline 15 & 30 & $17 \mathrm{~s}$ & $2^{-72}$ & $1595 \mathrm{~s}$ & 30 & $18410 \mathrm{~s}$ & & \\
\hline 16 & 32 & $18 \mathrm{~s}$ & & & 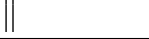 & & $\|$ & \\
\hline Total time & \multicolumn{2}{|c|}{$1039 \mathrm{~s}$} & \multicolumn{2}{|c|}{$28.29 \mathrm{~h}$} & \multicolumn{2}{|c|}{$11.95 \mathrm{~h}$} & \multicolumn{2}{|c|}{$53.2 \mathrm{~h}$} \\
\hline
\end{tabular}


Improving the MILP-based Security Evaluation Algorithm against Differential/Linear 456

Cryptanalysis Using A Divide-and-Conquer Approach

Table 4: Experimental results of RECTANGLE

\begin{tabular}{|c|c|c|c|c|c|c|c|c|c|}
\hline \multirow{2}{*}{ Rounds } & \multicolumn{4}{|c|}{ Differential cryptanalysis } & \multicolumn{5}{|c|}{ Linear cryptanalysis } \\
\hline & $\left.\# \# A S_{D}\right\}$ & Time & $\operatorname{Pr}_{D}$ & Time & $\|$ & $\#\left\{A S_{L}\right\}$ & Time & $\| \operatorname{Cor}_{L}$ & Time \\
\hline 1 & 1 & 1s & $2^{-2}$ & $1 \mathrm{~s}$ & & 1 & $1 \mathrm{~s}$ & $2^{-1}$ & $0 \mathrm{~s}$ \\
\hline 2 & 2 & $1 \mathrm{~s}$ & $2^{-4}$ & $1 \mathrm{~s}$ & & 2 & $1 \mathrm{~s}$ & $2^{-2}$ & $1 \mathrm{~s}$ \\
\hline 3 & 3 & $1 \mathrm{~s}$ & $2^{-7}$ & $8 s$ & & 3 & 1s & $2^{-4}$ & $5 \mathrm{~s}$ \\
\hline 4 & 4 & $2 \mathrm{~s}$ & $2^{-10}$ & $27 \mathrm{~s}$ & & 4 & $2 \mathrm{~s}$ & $2^{-6}$ & $9 \mathrm{~s}$ \\
\hline 5 & 6 & $11 \mathrm{~s}$ & $2^{-14}$ & $128 \mathrm{~s}$ & & 6 & $6 \mathrm{~s}$ & $2^{-8}$ & $41 \mathrm{~s}$ \\
\hline 6 & 8 & $13 \mathrm{~s}$ & $2^{-18}$ & $6 \mathrm{~s}$ & & 8 & $8 \mathrm{~s}$ & $2^{-10}$ & $6 \mathrm{~s}$ \\
\hline 7 & 11 & $11 \mathrm{~s}$ & $2^{-25}$ & $17 \mathrm{~s}$ & & 10 & $5 \mathrm{~s}$ & $2^{-13}$ & $15 \mathrm{~s}$ \\
\hline 8 & 13 & $11 \mathrm{~s}$ & $2^{-31}$ & $28 \mathrm{~s}$ & & 12 & $9 \mathrm{~s}$ & $2^{-16}$ & $24 \mathrm{~s}$ \\
\hline 9 & 15 & $11 \mathrm{~s}$ & $2^{-36}$ & $41 \mathrm{~s}$ & & 14 & $11 \mathrm{~s}$ & $2^{-19}$ & $78 \mathrm{~s}$ \\
\hline 10 & 17 & $25 \mathrm{~s}$ & $2^{-41}$ & $96 \mathrm{~s}$ & & 16 & $25 \mathrm{~s}$ & $2^{-22}$ & $260 \mathrm{~s}$ \\
\hline 11 & 19 & $47 \mathrm{~s}$ & $2^{-46}$ & $297 \mathrm{~s}$ & & 18 & $38 \mathrm{~s}$ & $2^{-25}$ & $1772 \mathrm{~s}$ \\
\hline 12 & 21 & $120 \mathrm{~s}$ & $2^{-51}$ & $669 \mathrm{~s}$ & & 20 & $131 \mathrm{~s}$ & $2^{-28}$ & $5927 \mathrm{~s}$ \\
\hline 13 & 23 & $597 \mathrm{~s}$ & $2^{-56}$ & 2798 s & & 22 & $428 \mathrm{~s}$ & $2^{-31}$ & $31491 \mathrm{~s}$ \\
\hline 14 & 25 & 2218 s & $2^{-61}$ & $12410 \mathrm{~s}$ & & 24 & $1615 \mathrm{~s}$ & $2^{-34}$ & $177473 \mathrm{~s}$ \\
\hline 15 & 27 & $12753 \mathrm{~s}$ & $2^{-66}$ & $40989 \mathrm{~s}$ & & 26 & $5588 \mathrm{~s}$ & & \\
\hline 16 & 29 & $36891 \mathrm{~s}$ & & & & 28 & $21352 \mathrm{~s}$ & & \\
\hline Total time & \multicolumn{2}{|c|}{$14.64 \mathrm{~h}$} & \multicolumn{2}{|c|}{$15.98 \mathrm{~h}$} & & \multicolumn{2}{|c|}{$8.12 \mathrm{~h}$} & \multicolumn{2}{|c|}{$60.31 \mathrm{~h}$} \\
\hline
\end{tabular}

\subsection{GIFT-64}

GIFT $\left[\mathrm{BPP}^{+}\right.$17] is an SPN cipher which is similar to PRESENT. It has two versions: GIFT64 and GIFT-128, whose block sizes are 64 bits and 128 bits respectively. In this paper, we focus on version GIFT-64. Both differential and linear branch numbers of GIFT-64 S-box are equal to 2. For GIFT-64, we obtain the minimum number of differentially/linearly active S-boxes for up to 16/15 rounds and find the best differential/linear characteristics for up to $15 / 13$ rounds.

The designers of GIFT gave a 9-round differential characteristic with the probability $2^{-44.415}$. Then in [ZDY19], Zhu et al. provided 9/12/13-round differential characteristics with probabilities $2^{-42} / 2^{-60} / 2^{-64}$ based on MILP technique. Recently, Li et al. [LWZZ19] found better $12 / 13$-round differential characteristics with probabilities $2^{-58} / 2^{-62}$ by using MILP technique. However, the differential characteristics they found are not proven the best ones, while we find the best differential characteristics covered from 1 to 15 rounds.

\subsection{RECTANGLE}

RECTANGLE is an SPN cipher proposed in $2015\left[\mathrm{ZBL}^{+} 15\right]$. Both differential and linear branch numbers of RECTANGLE S-box are equal to 2. For RECTANGLE, we obtain the minimum number of differentially/linearly active S-boxes for up to 16/16 rounds and find the best differential/linear characteristics for up to $15 / 14$ rounds.

The 64-bits plaintext of RECTANGLE is pictured as a rectangular array with 4 rows and 16 columns. The permutation of RECTANGLE is a left rotation to each row. If there is exactly 1 or 2 active S-boxes at a certain round, the index of the 1st active S-box has no influence on the minimum number of active S-boxes (or minimum weight) for $r$-round RECTANGLE. Therefore, different from the partition of the set of all possible $r$-round differential/linear characteristics for PRESENT and GIFT, we divide the set for RECTANGLE into $\left(1 \times\left(2^{4}-1\right) \times r+15 \times\left(2^{4}-1\right)^{2} \times r+1\right)$ smaller subsets. The number of resulting subsets for RECTANGLE is much less than those for PRESENT and GIFT.

\subsection{LBLOCK and TWINE}

LBLOCK [WZ11] and TWINE [SMMK12] are two similar Feistel ciphers, and both them are word-oriented ciphers. For LBLOCK, we obtain the minimum number of differen- 
Table 5: Experimental results of LBLOCK

\begin{tabular}{|c|c|c|c|c|c|c|c|c|}
\hline \multirow{2}{*}{ Rounds } & \multicolumn{4}{|c|}{ Differential cryptanalysis } & \multicolumn{4}{|c|}{ Linear cryptanalysis } \\
\hline & $\#\left\{A S_{D}\right\}$ & Time & $\| \operatorname{Pr}_{D}$ & Time & $\#\left\{A S_{L}\right\}$ & Time & $\| \operatorname{Cor}_{L}$ & Time \\
\hline 1 & 0 & $0 \mathrm{~s}$ & $2^{0}$ & $0 \mathrm{~s}$ & 0 & $0 \mathrm{~s}$ & $2^{-0}$ & $0 \mathrm{~s}$ \\
\hline 2 & 1 & 0s & $2^{-2}$ & 1s & 1 & 0s & $2^{-1}$ & Os \\
\hline 3 & 2 & $0 \mathrm{~s}$ & $2^{-4}$ & $0 \mathrm{~s}$ & 2 & $0 \mathrm{~s}$ & $2^{-2}$ & $0 \mathrm{~s}$ \\
\hline 4 & 3 & $1 \mathrm{~s}$ & $2^{-6}$ & $1 \mathrm{~s}$ & 3 & $0 \mathrm{~s}$ & $2^{-3}$ & $0 \mathrm{~s}$ \\
\hline 5 & 4 & $1 \mathrm{~s}$ & $2^{-8}$ & $1 \mathrm{~s}$ & 4 & $1 \mathrm{~s}$ & $2^{-4}$ & $1 \mathrm{~s}$ \\
\hline 6 & 6 & $1 \mathrm{~s}$ & $2^{-12}$ & $1 \mathrm{~s}$ & 6 & $1 \mathrm{~s}$ & $2^{-6}$ & $1 \mathrm{~s}$ \\
\hline 7 & 8 & $1 \mathrm{~s}$ & $2^{-16}$ & $1 \mathrm{~s}$ & 8 & $1 \mathrm{~s}$ & $2^{-8}$ & $2 \mathrm{~s}$ \\
\hline 8 & 11 & $2 \mathrm{~s}$ & $2^{-22}$ & $2 \mathrm{~s}$ & 11 & $2 \mathrm{~s}$ & $2^{-11}$ & $2 \mathrm{~s}$ \\
\hline 9 & 14 & $2 \mathrm{~s}$ & $2^{-28}$ & $2 \mathrm{~s}$ & 14 & $2 \mathrm{~s}$ & $2^{-14}$ & $2 \mathrm{~s}$ \\
\hline 10 & 18 & $6 \mathrm{~s}$ & $2^{-36}$ & $6 \mathrm{~s}$ & 18 & $6 \mathrm{~s}$ & $2^{-18}$ & $8 \mathrm{~s}$ \\
\hline 11 & 22 & $4 \mathrm{~s}$ & $2^{-44}$ & $4 \mathrm{~s}$ & 22 & $4 \mathrm{~s}$ & $2^{-22}$ & $4 \mathrm{~s}$ \\
\hline 12 & 24 & $5 \mathrm{~s}$ & $2^{-48}$ & $5 \mathrm{~s}$ & 24 & $6 s$ & $2^{-24}$ & $5 \mathrm{~s}$ \\
\hline 13 & 27 & $25 \mathrm{~s}$ & $2^{-56}$ & $812 \mathrm{~s}$ & 27 & $38 \mathrm{~s}$ & $2^{-27}$ & $2103 \mathrm{~s}$ \\
\hline 14 & 30 & $8 \mathrm{~s}$ & $2^{-62}$ & $848 \mathrm{~s}$ & 30 & $10 \mathrm{~s}$ & $2^{-30}$ & $15 \mathrm{~s}$ \\
\hline 15 & 32 & $19 \mathrm{~s}$ & $2^{-66}$ & $820 \mathrm{~s}$ & 32 & $28 \mathrm{~s}$ & $2^{-33}$ & $5669 \mathrm{~s}$ \\
\hline 16 & 35 & $30 \mathrm{~s}$ & $2^{-72}$ & $6002 \mathrm{~s}$ & 35 & $55 \mathrm{~s}$ & & \\
\hline 17 & 36 & $29 \mathrm{~s}$ & & & 36 & $31 \mathrm{~s}$ & & \\
\hline 18 & 39 & $10 \mathrm{~s}$ & & & 39 & $11 \mathrm{~s}$ & & \\
\hline 19 & 41 & $190 \mathrm{~s}$ & & & 41 & $6 s$ & & \\
\hline 20 & 44 & $1828 \mathrm{~s}$ & $\|$ & & 44 & $40 \mathrm{~s}$ & $\|$ & \\
\hline Total time & \multicolumn{2}{|c|}{$352 \mathrm{~s}$} & \multicolumn{2}{|c|}{$2.36 \mathrm{~h}$} & \multicolumn{2}{|c|}{$242 \mathrm{~s}$} & \multicolumn{2}{|c|}{$2.17 \mathrm{~h}$} \\
\hline
\end{tabular}

Table 6: Experimental results of TWINE

\begin{tabular}{|c|c|c|c|c|c|c|c|c|c|}
\hline \multirow{2}{*}{ Rounds } & \multicolumn{5}{|c|}{ Differential cryptanalysis } & \multicolumn{4}{|c|}{ Linear cryptanalysis } \\
\hline & $\|$ & $\#\left\{A S_{D}\right\}$ & Time & $\| \operatorname{Pr}_{D}$ & Time & $\| \#\left\{A S_{L}\right\}$ & Time & $\operatorname{Cor}_{L}$ & Time \\
\hline 1 & $\|$ & 0 & $0 \mathrm{~s}$ & $2^{0}$ & $0 \mathrm{~s}$ & 0 & $0 \mathrm{~s}$ & $2^{0}$ & $0 \mathrm{~s}$ \\
\hline 2 & & 1 & $0 \mathrm{~s}$ & $2^{-2}$ & $0 \mathrm{~s}$ & 1 & $0 \mathrm{~s}$ & $2^{-1}$ & $0 \mathrm{~s}$ \\
\hline 3 & & 2 & $0 \mathrm{~s}$ & $2^{-4}$ & $0 \mathrm{~s}$ & 2 & $0 \mathrm{~s}$ & $2^{-2}$ & $1 \mathrm{~s}$ \\
\hline 4 & & 3 & $0 \mathrm{~s}$ & $2^{-6}$ & $1 \mathrm{~s}$ & 3 & $1 \mathrm{~s}$ & $2^{-3}$ & $1 \mathrm{~s}$ \\
\hline 5 & & 4 & $1 \mathrm{~s}$ & $2^{-8}$ & $1 \mathrm{~s}$ & 4 & $1 \mathrm{~s}$ & $2^{-4}$ & $1 \mathrm{~s}$ \\
\hline 6 & & 6 & $1 \mathrm{~s}$ & $2^{-12}$ & $1 \mathrm{~s}$ & 6 & $1 \mathrm{~s}$ & $2^{-6}$ & $2 \mathrm{~s}$ \\
\hline 7 & & 8 & $1 \mathrm{~s}$ & $2^{-16}$ & $1 \mathrm{~s}$ & 8 & $2 \mathrm{~s}$ & $2^{-8}$ & $2 \mathrm{~s}$ \\
\hline 8 & & 11 & $1 \mathrm{~s}$ & $2^{-22}$ & $2 \mathrm{~s}$ & 11 & $3 \mathrm{~s}$ & $2^{-11}$ & $3 \mathrm{~s}$ \\
\hline 9 & & 14 & $2 \mathrm{~s}$ & $2^{-28}$ & $2 \mathrm{~s}$ & 14 & $3 \mathrm{~s}$ & $2^{-14}$ & $4 \mathrm{~s}$ \\
\hline 10 & & 18 & $6 \mathrm{~s}$ & $2^{-38}$ & $52 \mathrm{~s}$ & 18 & $10 \mathrm{~s}$ & $2^{-18}$ & $17 \mathrm{~s}$ \\
\hline 11 & & 22 & $4 \mathrm{~s}$ & $2^{-46}$ & $49 \mathrm{~s}$ & 22 & $4 \mathrm{~s}$ & $2^{-22}$ & $12 \mathrm{~s}$ \\
\hline 12 & & 24 & $4 \mathrm{~s}$ & $2^{-51}$ & $63 \mathrm{~s}$ & 24 & $6 s$ & $2^{-24}$ & $8 \mathrm{~s}$ \\
\hline 13 & & 27 & $24 \mathrm{~s}$ & $2^{-58}$ & $7905 \mathrm{~s}$ & 27 & $53 \mathrm{~s}$ & $2^{-27}$ & $364 \mathrm{~s}$ \\
\hline 14 & & 30 & $14 \mathrm{~s}$ & $2^{-64}$ & $17153 \mathrm{~s}$ & 30 & $12 \mathrm{~s}$ & $2^{-30}$ & $16 \mathrm{~s}$ \\
\hline 15 & & 32 & $14 \mathrm{~s}$ & $2^{-68}$ & 28840 s & 32 & $37 \mathrm{~s}$ & $2^{-32}$ & $261 \mathrm{~s}$ \\
\hline 16 & & 35 & $19 \mathrm{~s}$ & & & 35 & $49 \mathrm{~s}$ & $2^{-35}$ & $66 \mathrm{~s}$ \\
\hline 17 & & 36 & $17 \mathrm{~s}$ & & & 36 & $57 \mathrm{~s}$ & & \\
\hline 18 & & 39 & $9 \mathrm{~s}$ & & & 39 & $11 \mathrm{~s}$ & & \\
\hline 19 & & 41 & $5 \mathrm{~s}$ & & & 41 & $7 \mathrm{~s}$ & & \\
\hline 20 & & 44 & $17 \mathrm{~s}$ & $\|$ & & 44 & $42 \mathrm{~s}$ & & \\
\hline Total time & $\|$ & \multicolumn{2}{|c|}{$139 \mathrm{~s}$} & \multicolumn{2}{|c|}{$15.02 \mathrm{~h}$} & \multicolumn{2}{|c|}{$299 \mathrm{~s}$} & \multicolumn{2}{|c|}{$758 \mathrm{~s}$} \\
\hline
\end{tabular}


Improving the MILP-based Security Evaluation Algorithm against Differential/Linear 458

Cryptanalysis Using A Divide-and-Conquer Approach

tially/linearly active S-boxes for up to 20/20 rounds and find the best differential/linear characteristics for up to 16/15 rounds. For TWINE, we obtain the minimum number of differentially/linearly active S-boxes for up to 20/20 rounds and find the best differential/linear characteristics for up to $15 / 16$ rounds.

\section{Conclusion}

In this paper, we propose a new MILP-based search algorithm for the security evaluation against differential/linear cryptanalysis by incorporating the idea of a divide-and-conquer approach. For the search for an $r$-round block cipher, we first divide the set of all possible $r$-round differential/linear characteristics into several subsets, then separately search each subset; we also use several techniques to early terminate the search of a subset, which improves efficiency remarkably; finally the optimal solutions within smaller subsets are combined to give the optimal solution within the whole set. As a result, we obtain a more efficient search algorithm.

We only apply our new algorithm to five lightweight block ciphers in this paper. We point out that the permutation layers of these five ciphers are all bit permutations. For each of five ciphers, the best differential and linear characteristics we found have a low number $(0,1$ or 2$)$ of active S-boxes at a certain round. In future work, we will consider applying our algorithm to the ciphers with stronger permutation layer, such as AES [DR02], NOEKEON [DPAR00], SERPENT [BAK98], etc. In Tables 2-6, although the weights of the best differential/linear characteristics for some reduced rounds are larger than cipher's block size $n$ /half of $n$, we argue that it is possibly useful when the differential/linear clustering is taken into consideration [DR02, Nyb94]. We explain how to search for relatedkey differential characteristics [Bih93] in Appendix B, but it seems to be more difficult and requires more work. We leave these researches as our future work.

\section{Acknowledgments}

The authors would like to thank the anonymous reviewers for their helpful comments. This work was supported by the National Natural Science Foundation of China (No.61379138).

\section{References}

$\left[\mathrm{AST}^{+}\right.$17] Ahmed Abdelkhalek, Yu Sasaki, Yosuke Todo, Mohamed Tolba, and Amr M. Youssef. MILP Modeling for (Large) S-boxes to Optimize Probability of Differential Characteristics. IACR Trans. Symmetric Cryptol., 2017(4):99129, 2017.

[BAK98] Eli Biham, Ross J. Anderson, and Lars R. Knudsen. Serpent: A New Block Cipher Proposal. In Fast Software Encryption, 5th International Workshop, FSE '98, Paris, France, March 23-25, 1998, Proceedings, pages 222-238, 1998.

[Bih93] Eli Biham. New Types of Cryptanalytic Attacks Using related Keys. In Advances in Cryptology - EUROCRYPT'93, Workshop on the Theory and Application of of Cryptographic Techniques, Lofthus, Norway, May 23-27, 1993, Proceedings, pages 398-409, 1993.

[BJK $\left.{ }^{+} 16\right]$ Christof Beierle, Jérémy Jean, Stefan Kölbl, Gregor Leander, Amir Moradi, Thomas Peyrin, Yu Sasaki, Pascal Sasdrich, and Siang Meng Sim. The SKINNY Family of Block Ciphers and Its Low-Latency Variant MANTIS. In 
Advances in Cryptology - CRYPTO 2016 - 36th Annual International Cryptology Conference, Santa Barbara, CA, USA, August 14-18, 2016, Proceedings, Part II, pages 123-153, 2016.

$\left[\mathrm{BKL}^{+} 07\right]$ Andrey Bogdanov, Lars R. Knudsen, Gregor Leander, Christof Paar, Axel Poschmann, Matthew J. B. Robshaw, Yannick Seurin, and C. Vikkelsoe. PRESENT: An Ultra-Lightweight Block Cipher. In Cryptographic Hardware and Embedded Systems - CHES 2007, 9th International Workshop, Vienna, Austria, September 10-13, 2007, Proceedings, pages 450-466, 2007.

$\left[\mathrm{BPP}^{+} 17\right]$ Subhadeep Banik, Sumit Kumar Pandey, Thomas Peyrin, Yu Sasaki, Siang Meng Sim, and Yosuke Todo. GIFT: A Small Present - Towards Reaching the Limit of Lightweight Encryption. In Cryptographic Hardware and Embedded Systems - CHES 2017 - 19th International Conference, Taipei, Taiwan, September 25-28, 2017, Proceedings, pages 321-345, 2017.

[BS91] Eli Biham and Adi Shamir. Differential Cryptanalysis of DES-like Cryptosystems. J. Cryptology, 4(1):3-72, 1991.

[BSS $\left.{ }^{+} 13\right] \quad$ Ray Beaulieu, Douglas Shors, Jason Smith, Stefan Treatman-Clark, Bryan Weeks, and Louis Wingers. The SIMON and SPECK Families of Lightweight Block Ciphers. Cryptology ePrint Archive, Report 2013/404, 2013. https: //eprint.iacr.org/2013/404.

[DPAR00] Joan Daemen, Michaël Peeters, Gilles Van Assche, and Vincent Rijmen. Nessie Proposal: NOEKEON. 2000. http://gro.noekeon.org.

[DR02] Joan Daemen and Vincent Rijmen. The Design of Rijndael: AES - The Advanced Encryption Standard. Information Security and Cryptography. Springer, 2002.

$\left[\mathrm{FWG}^{+} 16\right]$ Kai Fu, Meiqin Wang, Yinghua Guo, Siwei Sun, and Lei Hu. MILP-Based Automatic Search Algorithms for Differential and Linear Trails for Speck. In Fast Software Encryption - 23rd International Conference, FSE 2016, Bochum, Germany, March 20-23, 2016, Revised Selected Papers, pages 268-288, 2016.

[Gur] http://www.gurobi.com.

[LWZZ19] Lingchen Li, Wenling Wu, Yafei Zheng, and Lei Zhang. The Relationship between the Construction and Solution of the MILP Models and Applications. Cryptology ePrint Archive, Report 2019/049, 2019. https://eprint.iacr. org/2019/049.

[Mat93] Mitsuru Matsui. Linear Cryptanalysis Method for DES Cipher. In Advances in Cryptology - EUROCRYPT' 93, Workshop on the Theory and Application of of Cryptographic Techniques, Lofthus, Norway, May 23-27, 1993, Proceedings, pages 386-397, 1993.

[Mat94] Mitsuru Matsui. On Correlation Between the Order of S-boxes and the Strength of DES. In Advances in Cryptology - EUROCRYPT '94, Workshop on the Theory and Application of Cryptographic Techniques, Perugia, Italy, May 9-12, 1994, Proceedings, pages 366-375, 1994.

[MWGP11] Nicky Mouha, Qingju Wang, Dawu Gu, and Bart Preneel. Differential and Linear Cryptanalysis Using Mixed-Integer Linear Programming. In Information Security and Cryptology - 7th International Conference, Inscrypt 2011, Beijing, China, November 30 - December 3, 2011. Revised Selected Papers, pages 57-76, 2011. 
[Nyb94] Kaisa Nyberg. Linear Approximation of Block Ciphers. In Advances in Cryptology - EUROCRYPT' '94, Workshop on the Theory and Application of Cryptographic Techniques, Perugia, Italy, May 9-12, 1994, Proceedings, pages 439-444, 1994.

[sag] http://www.sagemath.org.

$\left[\mathrm{SHQ}^{+} 15\right]$ Siwei Sun, Lei Hu, Kexin Qiao, Xiaoshuang Ma, Jinyong Shan, and Ling Song. Improvement on the Method for Automatic Differential Analysis and Its Application to Two Lightweight Block Ciphers DESL and LBlock-s. In Advances in Information and Computer Security - 10th International Workshop on Security, IWSEC 2015, Nara, Japan, August 26-28, 2015, Proceedings, pages $97-111,2015$.

$\left[\mathrm{SHS}^{+} 13\right]$ Siwei Sun, Lei Hu, Ling Song, Yonghong Xie, and Peng Wang. Automatic Security Evaluation of Block Ciphers with S-bP Structures Against RelatedKey Differential Attacks. In Information Security and Cryptology - 9th International Conference, Inscrypt 2013, Guangzhou, China, November 27-30, 2013, Revised Selected Papers, pages 39-51, 2013.

$\left[\mathrm{SHW}^{+} 14 \mathrm{a}\right]$ Siwei Sun, Lei Hu, Meiqin Wang, Peng Wang, Kexin Qiao, Xiaoshuang Ma, Danping Shi, Ling Song, and Kai Fu. Towards Finding the Best Characteristics of Some Bit-oriented Block Ciphers and Automatic Enumeration of (Related-key) Differential and Linear Characteristics with Predefined Properties. Cryptology ePrint Archive, Report 2014/747, 2014. https://eprint.iacr.org/2014/747.

$\left[\mathrm{SHW}^{+} 14 \mathrm{~b}\right]$ Siwei Sun, Lei Hu, Peng Wang, Kexin Qiao, Xiaoshuang Ma, and Ling Song. Automatic Security Evaluation and (Related-key) Differential Characteristic Search: Application to SIMON, PRESENT, LBlock, DES(L) and Other Bit-Oriented Block Ciphers. In Advances in Cryptology - ASIACRYPT 2014 - 20th International Conference on the Theory and Application of Cryptology and Information Security, Kaoshiung, Taiwan, R.O.C., December 7-11, 2014. Proceedings, Part I, pages 158-178, 2014.

[SMMK12] Tomoyasu Suzaki, Kazuhiko Minematsu, Sumio Morioka, and Eita Kobayashi. TWINE: A Lightweight Block Cipher for Multiple Platforms. In Selected Areas in Cryptography, 19th International Conference, SAC 2012, Windsor, ON, Canada, August 15-16, 2012, Revised Selected Papers, pages 339-354, 2012.

[ST17] Yu Sasaki and Yosuke Todo. New Algorithm for Modeling S-box in MILP Based Differential and Division Trail Search. In Innovative Security Solutions for Information Technology and Communications - 10th International Conference, SecITC 2017, Bucharest, Romania, June 8-9, 2017, Revised Selected Papers, pages 150-165, 2017.

[Wan08] Meiqin Wang. Differential Cryptanalysis of Reduced-Round PRESENT. In Progress in Cryptology - AFRICACRYPT 2008, First International Conference on Cryptology in Africa, Casablanca, Morocco, June 11-14, 2008. Proceedings, pages 40-49, 2008.

[WSTP12] Meiqin Wang, Yue Sun, Elmar Tischhauser, and Bart Preneel. A Model for Structure Attacks, with Applications to PRESENT and Serpent. In Fast Software Encryption - 19th International Workshop, FSE 2012, Washington, DC, USA, March 19-21, 2012. Revised Selected Papers, pages 49-68, 2012. 
[WW11] Shengbao Wu and Mingsheng Wang. Security evaluation against differential cryptanalysis for block cipher structures. Cryptology ePrint Archive, Report 2011/551, 2011. https://eprint.iacr.org/2011/551.

[WZ11] Wenling Wu and Lei Zhang. LBlock: A Lightweight Block Cipher. In Applied Cryptography and Network Security - 9th International Conference, ACNS 2011, Nerja, Spain, June 7-10, 2011. Proceedings, pages 327-344, 2011.

$\left[\mathrm{ZBL}^{+}{ }^{15}\right]$ Wentao Zhang, Zhenzhen Bao, Dongdai Lin, Vincent Rijmen, Bohan Yang, and Ingrid Verbauwhede. RECTANGLE: a bit-slice lightweight block cipher suitable for multiple platforms. SCIENCE CHINA Information Sciences, 58(12):1-15, 2015.

[ZDY19] Baoyu Zhu, Xiaoyang Dong, and Hongbo Yu. MILP-Based Differential Attack on Round-Reduced GIFT. In Topics in Cryptology - CT-RSA 2019 - The Cryptographers' Track at the RSA Conference 2019, San Francisco, CA, USA, March 4-8, 2019, Proceedings, pages 372-390, 2019.

[ZSCH18] Yingjie Zhang, Siwei Sun, Jiahao Cai, and Lei Hu. Speeding up MILP aided differential characteristic search with matsui's strategy. In Information Security - 21st International Conference, ISC 2018, Guildford, UK, September 9-12, 2018, Proceedings, pages 101-115, 2018. 


\section{A Search Algorithm for Feistel Ciphers}

In this section, we evaluate the security of Feistel ciphers against differential/linear cryptanalysis by using the idea of a divide-and-conquer approach.

Dividing the Set of All Possible Differential/Linear Characteristics. For Feistel ciphers, the differential/linear characteristics with the highest probability/absolute linear bias are likely to have no active S-box at a certain round. Therefore, we first divide the set of all possible $r$-round differential/linear characteristics for an $r$-round Feistel cipher into:

Subset-0 In this kind of subset, differential/linear characteristics have no active S-box at a certain round;

Subset-1 In this subset, differential/linear characteristics have at least one active S-box in each round.

For Subset-0, we further divide it by fixing the index $i(i=1,2, \ldots, r)$, such that round $i$ contains no active S-box. The subsets divided now are not small enough, thus we further divide the set by traversing the difference/linear mask patterns of the S-boxes at the $(i+1)$ th round. The reason why we don't divide the set by traversing the input difference of round $i$ is that in this partition, the number of resulting subsets will be too large.

Similar to the case for SPN ciphers, we use $\mathcal{D}_{r, N_{A}, i, P}$ to denote the subset of $r$-round differential/linear characteristics that satisfy the following two constraints:

Constraint 1 there are at least $N_{A}$ active S-boxes in each round except for round $i$;

Constraint 2 the difference/linear mask patterns of the S-boxes at round $i$ and $(i+1)$ are equal to zeros and $P$ respectively.

When $i=0$, there is no constraint on the difference/linear mask patterns, and we ignore the second constraint by (not rigorously) writing $P=0$. Differential/linear characteristics in the subset are only required to satisfy the Constraint 1, i.e., there are at least $N_{A}$ active S-boxes in each round. And if $i=r$, based on the Feistel structure, we regard the constraint of "difference/linear mask patterns of the S-boxes at round $(i+1)$ equal $P$ " as the constraint of "difference/linear mask patterns of the S-boxes at round $(i-1)$ are determined by $P$ ".

In summary, the set of all possible $r$-round differential/linear characteristics for an $r$-round Feistel cipher is divided into the following subsets:

$$
\left(\bigcup_{i, P} \mathcal{D}_{r, 0, i, P}\right) \bigcup \mathcal{D}_{r, 1,0,0}
$$

where $i \in\{1,2, \ldots, r\}, P$ belongs to the set of all possible difference/linear mask patterns of the S-boxes at round $(i+1)$. Take LBLOCK as an example, its block size is 64 bits and it uses a $4 \times 4 \mathrm{~S}$-box. Thus it applied $8 \mathrm{~S}$-boxes in each round. If round $i$ has no active S-box, there is at least one active S-box at round $(i+1)$. Based on Equation (14), the set of all possible differential/linear characteristic for $r$-round LBLOCK is divided into $\left(r \times\left(2^{8}-1\right)+1\right)$ subsets.

Building MILP Models for Searching Subsets. For a subset divided in Equation (14), we build an MILP model to search it. Let $A_{j, k}$ denote the word-level input difference of the $k$ th S-box at round $j$, Constraint 1 is described by Equation (7), and Constraint 2 is described by:

$$
\left\{\begin{array}{l}
A_{i, k}=0, k \in\left\{0,1, \ldots, N_{S}-1\right\} \\
A_{i+1, k}=p_{k}, k \in\left\{0,1, \ldots, N_{S}-1\right\},
\end{array}\right.
$$


where $N_{S}$ is the total number of S-boxes in each round, and $P$ is expressed as $P=$ $\left(p_{0}, p_{1}, \ldots, p_{N_{S}-1}\right)$. By using Sun et al.'s model framework $\left[\mathrm{SHW}^{+} 14 \mathrm{~b}, \mathrm{SHW}^{+} 14 \mathrm{a}\right]$ and Equations $(7,15)$, we build an MILP model whose feasible region is the subset.

Search Algorithm for Feistel Ciphers. For a subset named $\mathcal{D}_{r, N_{A}, i, P}$, we use $\mathrm{LB}\left[r, N_{A}, i, P\right]$ to store a lower bound of the minimum weight (or number of active S-boxes) of the differential/linear characteristics within it. Techniques for the improvement and methods for calculating lower bounds introduced for SPN ciphers are also used for Feistel ciphers. It should be noted that for the subset $\mathcal{D}_{r, 0, i, P}$ with $i \neq 0, P \neq 0$, difference patterns of the S-boxes at round $i$ equal zeros, but the input difference of round $i$ is indeterminate. Therefore, the sum of the minimum weights (or numbers of active S-boxes) of the differential/linear characteristics within $\mathcal{D}_{i, 0, i, P}$ and $\mathcal{D}_{r-i+1,0,1, P}$ is smaller than or equal to the minimum weight (or number of active S-boxes) of the differential/linear characteristics within $\mathcal{D}_{r, 0, i, P}$. To obtain the minimum weight (or number of active S-boxes) of the differential/linear characteristics within $\mathcal{D}_{r, 0, i, P}$, we need to solve an MILP model whose feasible region is $\mathcal{D}_{r, 0, i, P}$. The process of searching for the best differential characteristics for Feistel ciphers is illustrated in Algorithm 2.

\section{B Security Evaluation against Related-Key Differential Crypt- analysis}

In this section, we extend our search algorithm to search for the optimal related-key differential characteristic. We observe that the related-key differential characteristics with the minimum number of active S-boxes or with the maximum differential probability are likely to have no active S-box at a certain round. Therefore, we use the partition method introduced for Feistel ciphers (see Appendix A) to divide the set of all possible related-key differential characteristics for an $r$-round cipher. In $\left[\mathrm{SHS}^{+} 13, \mathrm{SHW}^{+} 14 \mathrm{~b}, \mathrm{SHW}^{+} 14 \mathrm{a}\right]$, the authors described the differential behaviour of the key schedule and introduced the related-key model. The model can be used to both calculate the minimum number of related-key differentially active S-boxes and search for the best related-key differential characteristic. By using previous work, we build a related-key model to search each of the subsets divided. We point out that the search of a related-key subset is more difficult than the search of a single-key subset. This is mainly because in a related-key subset, there are plenty of possibilities of round keys. Besides, a related-key model is more difficult to be solved than a single-key model because the size of the related-key model is larger. Applying our algorithm to related-key differential cryptanalysis seems to require a large calculation, thus more techniques are needed to improve efficiency. A method to shorten the running time is to interrupt the solving process of a model at some point when the model is proven to have no better solution than the obtained currently best one (it can be done with Gurobi software). Moreover, if good solutions are found by searching a reduced number of subsets, the remaining subsets can be discarded. By doing this, good solutions rather than the best ones are obtained in a shorter time.

\section{Number of MILP Models Solved in Our Algorithm}

In previous MILP-based work, a problem is transformed into an MILP model. While in our search algorithm, a problem is solved by solving several MILP models that are easier to be solved. In this section, we study the number of models to be solved in our search algorithm.

Take searching for the best differential characteristics for the first 9-round PRESENT as an example, we list the number of models that have been solved in Table 7 . PRESENT 
Improving the MILP-based Security Evaluation Algorithm against Differential/Linear 464 Cryptanalysis Using A Divide-and-Conquer Approach

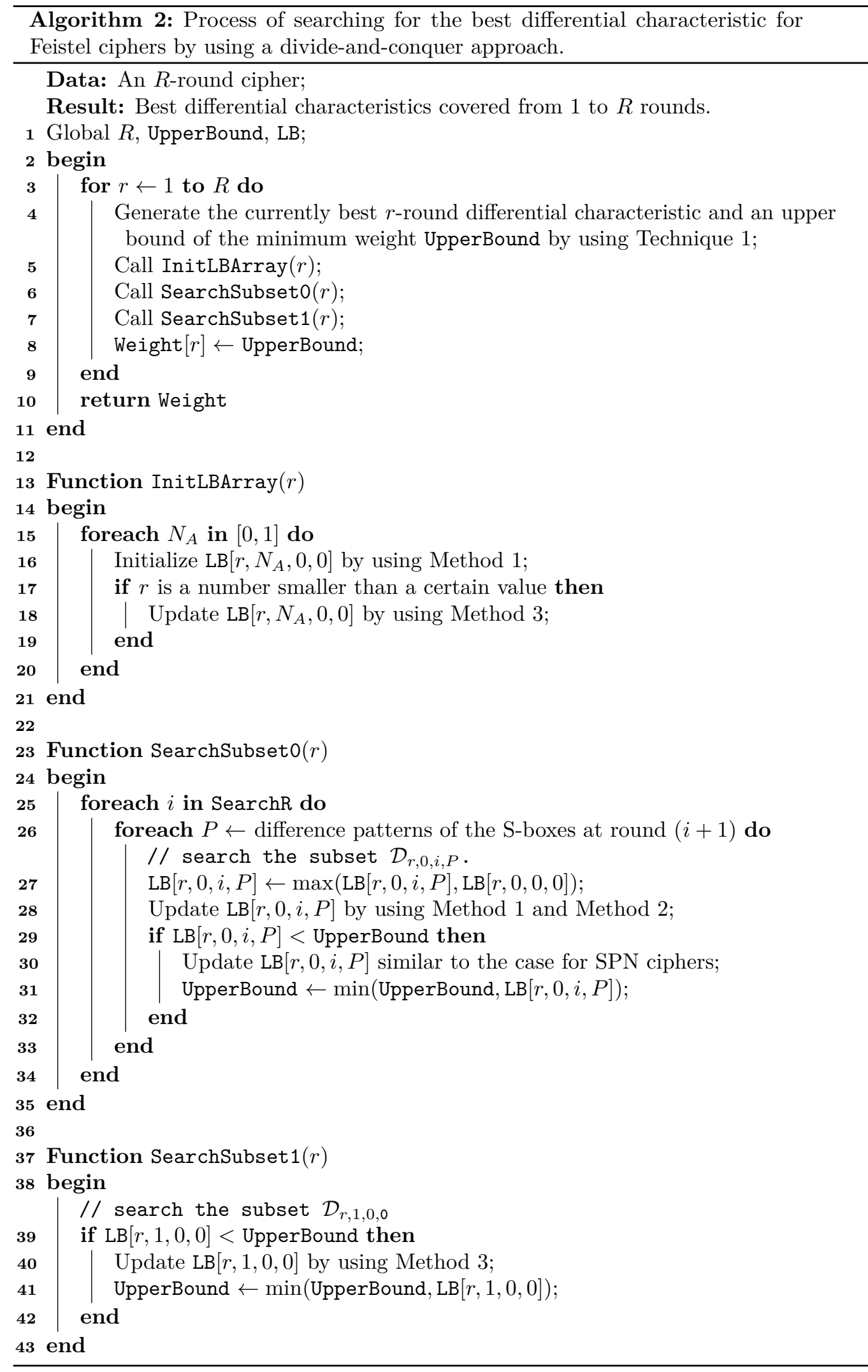


Table 7: Number of MILP models we solved for searching for the best differential characteristics for $r$-round PRESENT by using our algorithm

\begin{tabular}{|c|c|c|c|c|c|c|c|c|c|}
\hline \multirow{2}{*}{$r$} & \multicolumn{9}{|c|}{$r_{1}$ (number of rounds of the model built for) } \\
\hline & 1 & 2 & 3 & 4 & 5 & 6 & 7 & 8 & 9 \\
\hline 1 & $3+1$ & & & & & & & & \\
\hline 2 & $0+0$ & $3+0$ & & & & & & & \\
\hline 3 & $0+0$ & $0+0$ & $3+0$ & & & & & & \\
\hline 4 & $0+0$ & $0+0$ & $0+0$ & $3+0$ & & & & & \\
\hline 5 & $0+0$ & $0+0$ & $0+0$ & $0+0$ & $3+0$ & & & & \\
\hline 6 & $11958+108$ & $888+63$ & $108+8$ & $0+0$ & $0+0$ & $3+0$ & & & \\
\hline 7 & $0+0$ & $0+0$ & $0+0$ & $0+0$ & $0+0$ & $0+0$ & $3+0$ & & \\
\hline 8 & $0+0$ & $0+0$ & $0+0$ & $0+0$ & $0+0$ & $0+0$ & $0+0$ & $3+0$ & \\
\hline 9 & $0+0$ & $0+0$ & $0+0$ & $0+0$ & $0+0$ & $0+0$ & $0+0$ & $0+0$ & $3+0$ \\
\hline
\end{tabular}

The item "*+*" denotes the number of "Rough" models adding the number of "Tightest" models (introduced in Method 3 in Section3.4).

is a bit-oriented block cipher, and its differential branch number of an S-box is equal to 3. In our algorithm, "Rough" models and "Tightest" models (introduced in Method 3 in Section3.4) are used to estimate lower bounds of the minimum weights of the differential characteristics within the subsets divided. From Table 7 we see that, to search for the best differential characteristics for $r$-round PRESENT, several MILP models are solved. These models are built for $r_{1}$ rounds, $1 \leq r_{1} \leq r$, and most of them are easy to be solved.

For the search for $r=1$ rounds, we obtain the best 1-round differential characteristic by solving one "Tightest" model built for 1 round. Moreover, we solve three "Rough" models whose feasible regions are $\mathcal{D}_{1, N_{A}, 0,0}$ to assign values to $\operatorname{LB}\left[r, N_{A}, 0,0\right], N_{A}=1,2,3$.

For the search for $r=2,3,4,5,7,8,9$ rounds, the currently best $r$-round differential characteristic generated by using Technique 1 is exactly the best one. Three "Rough" models built for $r$ rounds are solved to assign values to $\operatorname{LB}\left[r, N_{A}, 0,0\right], N_{A}=1,2,3$. When searching an arbitrary subset, the lower bound of the minimum weight of the differential characteristics within the subset, namely, the value stored in the corresponding lower bound array is greater than or equal to the weight of the currently best $r$-round differential characteristic. Therefore, no other model is solved.

For the search for $r=6$ rounds, the currently best $r$-round differential characteristic generated by using Technique 1 is not the best one. Except for the three "Rough" models built for $r$ rounds are solved to assign values to $\mathrm{LB}\left[r, N_{A}, 0,0\right], N_{A}=1,2,3$, several "Rough" and "Tightest" models built for $r_{1}$ rounds are solved to search the subsets divided, $1 \leq r_{1} \leq r$.

\section{Examples of Best Differential and Linear Characteristics}

We provide the best differential and linear characteristics searched by implementing our search algorithm. 
Improving the MILP-based Security Evaluation Algorithm against Differential/Linear 466

Cryptanalysis Using A Divide-and-Conquer Approach

Table 8: The best differential characteristic with probability $2^{-78}$ for 18 -round PRESENT.

\begin{tabular}{c|c|c|c}
\hline Rounds & input difference of S-boxes & output difference of S-boxes & probability \\
\hline $1^{t h}$ & 0x0000000000001001 & 0x0000000000009009 & $2^{-4}$ \\
$2^{t h}$ & 0x0009000000000009 & 0x0004000000000004 & $2^{-4}$ \\
$3^{t h}$ & 0x0000100100000000 & 0x0000300300000000 & $2^{-4}$ \\
$4^{t h}$ & 0x0000000009000900 & 0x0000000004000400 & $2^{-4}$ \\
$5^{t h}$ & 0x0000004400000000 & 0x0000005500000000 & $2^{-4}$ \\
$6^{t h}$ & 0x0000030000000300 & 0x0000010000000100 & $2^{-6}$ \\
$7^{t h}$ & 0x0000000000000404 & 0x0000000000000505 & $2^{-4}$ \\
$8^{t h}$ & 0x0000000500000005 & 0x0000000100000001 & $2^{-6}$ \\
$9^{t h}$ & 0x0000000000000101 & 0x0000000000000909 & $2^{-4}$ \\
$10^{t h}$ & 0x0005000000000005 & 0x0001000000000001 & $2^{-6}$ \\
$11^{t h}$ & 0x0000000000001001 & 0x0000000000009009 & $2^{-4}$ \\
$12^{t h}$ & 0x0009000000000009 & 0x0004000000000004 & $2^{-4}$ \\
$13^{t h}$ & 0x0000100100000000 & 0x0000900900000000 & $2^{-4}$ \\
$14^{t h}$ & 0x0900000000000900 & 0x0400000000000400 & $2^{-4}$ \\
$15^{t h}$ & 0x0000400400000000 & 0x0000500500000000 & $2^{-4}$ \\
$16^{t h}$ & 0x0000090000000900 & 0x0000040000000400 & $2^{-4}$ \\
$17^{t h}$ & 0x0000040400000000 & 0x0000050500000000 & $2^{-4}$ \\
$18^{t h}$ & 0x0000050000000500 & 0x00000c0000000c00 & $2^{-4}$ \\
\hline
\end{tabular}

Table 9: The best linear characteristic with correlation $2^{-34}$ for 18 -round PRESENT.

\begin{tabular}{c|c|c|c}
\hline Rounds & input mask of S-boxes & output mask of S-boxes & correlation \\
\hline $1^{t h}$ & 0x0d00000000000000 & 0x0200000000000000 & $2^{-1}$ \\
$2^{t h}$ & 0x0000000040000000 & 0x0000000040000000 & $2^{-2}$ \\
$3^{t h}$ & 0x0000008000000000 & 0x0000002000000000 & $2^{-2}$ \\
$4^{t h}$ & 0x0000000002000000 & 0x0000000004000000 & $2^{-2}$ \\
$5^{t h}$ & 0x0000004000000000 & 0x0000008000000000 & $2^{-2}$ \\
$6^{t h}$ & 0x0200000000000000 & 0x0400000000000000 & $2^{-2}$ \\
$7^{t h}$ & 0x0000400000000000 & 0x0000200000000000 & $2^{-2}$ \\
$8^{t h}$ & 0x0000000008000000 & 0x0000000002000000 & $2^{-2}$ \\
$9^{t h}$ & 0x0000000000400000 & 0x0000000000400000 & $2^{-2}$ \\
$10^{t h}$ & 0x0000002000000000 & 0x0000004000000000 & $2^{-2}$ \\
$11^{t h}$ & 0x0000020000000000 & 0x0000020000000000 & $2^{-2}$ \\
$12^{t h}$ & 0x0000000004000000 & 0x0000000002000000 & $2^{-2}$ \\
$13^{t h}$ & 0x0000000000400000 & 0x0000000000200000 & $2^{-2}$ \\
$14^{t h}$ & 0x0000000000200000 & 0x0000000000200000 & $2^{-2}$ \\
$15^{t h}$ & 0x0000000000200000 & 0x0000000000800000 & $2^{-2}$ \\
$16^{t h}$ & 0x0020000000000000 & 0x0020000000000000 & $2^{-2}$ \\
$17^{t h}$ & 0x0000000020000000 & 0x0000000080000000 & $2^{-2}$ \\
$18^{t h}$ & 0x0080000000000000 & 0x00f0000000000000 & $2^{-1}$ \\
\hline
\end{tabular}


Table 10: The best differential characteristic with probability $2^{-72}$ for 15 -round GIFT-64

\begin{tabular}{c|c|c|c}
\hline Rounds & input difference of S-boxes & output difference of S-boxes & probability \\
\hline $1^{t h}$ & 0x000f0000000c0000 & 0x000400000004000 & $2^{-4}$ \\
$2^{t h}$ & 0x0000404000000000 & 0x000050500000000 & $2^{-4}$ \\
$3^{t h}$ & 0x0500000005000000 & 0x020000000200000 & $2^{-6}$ \\
$4^{t h}$ & 0x2020000000000000 & 0x505000000000000 & $2^{-4}$ \\
$5^{t h}$ & 0x5000000050000000 & 0x200000002000000 & $2^{-6}$ \\
$6^{t h}$ & 0x0000202000000000 & 0x000050500000000 & $2^{-4}$ \\
$7^{t h}$ & 0x0500000005000000 & 0x020000000200000 & $2^{-6}$ \\
$8^{t h}$ & 0x2020000000000000 & 0x505000000000000 & $2^{-4}$ \\
$9^{t h}$ & 0x5000000050000000 & 0x200000002000000 & $2^{-6}$ \\
$10^{t h}$ & 0x0000202000000000 & 0x000050500000000 & $2^{-4}$ \\
$11^{t h}$ & 0x0500000005000000 & 0x020000000200000 & $2^{-6}$ \\
$12^{t h}$ & 0x2020000000000000 & 0x505000000000000 & $2^{-4}$ \\
$13^{t h}$ & 0x5000000050000000 & 0x200000002000000 & $2^{-6}$ \\
$14^{t h}$ & 0x0000202000000000 & 0x000050500000000 & $2^{-4}$ \\
$15^{t h}$ & 0x0500000005000000 & 0x0f0000000f000000 & $2^{-4}$ \\
\hline
\end{tabular}

Table 11: The best linear characteristic with correlation $2^{-34}$ for 13 -round GIFT-64

\begin{tabular}{c|c|c|c}
\hline Rounds & input mask of S-boxes & output mask of S-boxes & correlation \\
\hline $1^{t h}$ & 0x0c0c000000000000 & 0x0101000000000000 & $2^{-2}$ \\
$2^{t h}$ & 0x0000100000001000 & 0x0000800000008000 & $2^{-2}$ \\
$3^{t h}$ & 0x0000000000000808 & 0x0000000000000505 & $2^{-2}$ \\
$4^{t h}$ & 0x0000000500000005 & 0x0000000a0000000a & $2^{-2}$ \\
$5^{t h}$ & 0x0808000002020000 & 0x0505000005050000 & $2^{-4}$ \\
$6^{t h}$ & 0x0000505000005050 & 0x0000a0a00000a0a0 & $2^{-4}$ \\
$7^{t h}$ & 0x00000a0a00000a0a & 0x0000020800000208 & $2^{-6}$ \\
$8^{t h}$ & 0x0a0a000000000000 & 0x0208000000000000 & $2^{-3}$ \\
$9^{t h}$ & 0xa000000000000000 & 0x2000000000000000 & $2^{-1}$ \\
$10^{t h}$ & 0x0000200000000000 & 0x0000800000000000 & $2^{-2}$ \\
$11^{t h}$ & 0x0000000000000800 & 0x0000000000000500 & $2^{-1}$ \\
$12^{t h}$ & 0x0000000100000004 & 0x0000000800000006 & $2^{-2}$ \\
$13^{t h}$ & 0x0800000400020000 & 0x0500000600050000 & $2^{-3}$ \\
\hline
\end{tabular}

Table 12: The best differential characteristic with probability $2^{-66}$ for 15 -round RECTANGLE.

\begin{tabular}{c|c|c|c}
\hline Rounds & input difference of S-boxes & output difference of S-boxes & probability \\
\hline $1^{t h}$ & 0x0000f00009000000 & 0x0000200006000000 & $2^{-4}$ \\
$2^{t h}$ & 0x0000000060000200 & 0x0000000020000600 & $2^{-5}$ \\
$3^{t h}$ & 0x0200000000006000 & 0x0600000000002000 & $2^{-5}$ \\
$4^{t h}$ & 0x6000020000000000 & 0x2000060000000000 & $2^{-5}$ \\
$5^{t h}$ & 0x0000600002000000 & 0x0000200006000000 & $2^{-5}$ \\
$6^{t h}$ & 0x0000000060000200 & 0x0000000020000600 & $2^{-5}$ \\
$7^{t h}$ & 0x0200000000006000 & 0x0600000000002000 & $2^{-5}$ \\
$8^{t h}$ & 0x6000020000000000 & 0x2000060000000000 & $2^{-5}$ \\
$9^{t h}$ & 0xa000060000200000 & 0x0000200006000000 & $2^{-5}$ \\
$10^{t h}$ & 0x0000000060000200 & 0x0000000020000600 & $2^{-5}$ \\
$11^{t h}$ & 0x0000000000006800 & 0x0000000000002100 & $2^{-5}$ \\
$12^{t h}$ & 0x3000000000000000 & 0x8000000000000000 & $2^{-3}$ \\
$13^{t h}$ & 0x8000000000000000 & 0x1000000000000000 & $2^{-3}$ \\
$14^{t h}$ & 0x0001000000000000 & 0x0006000000000000 & $2^{-2}$ \\
$15^{t h}$ & 0x0040000200000000 & 0x00f0000d00000000 & $2^{-4}$ \\
\hline
\end{tabular}


Improving the MILP-based Security Evaluation Algorithm against Differential/Linear 468

Cryptanalysis Using A Divide-and-Conquer Approach

Table 13: The best linear characteristic with correlation $2^{-34}$ for 14-round RECTANGLE.

\begin{tabular}{c|c|c|c}
\hline Rounds & input mask of S-boxes & out mask of S-boxes & correlation \\
\hline $1^{t h}$ & 0x000f0000dc000000 & 0x0002000021000000 & $2^{-3}$ \\
$2^{t h}$ & 0x0000000200003000 & 0x0000000a00004000 & $2^{-2}$ \\
$3^{t h}$ & 0x0000000800060000 & 0x00000003000c0000 & $2^{-2}$ \\
$4^{t h}$ & 0x00000000005a0000 & 0x0000000000850000 & $2^{-4}$ \\
$5^{t h}$ & 0x0000000000c00010 & 0x0000000000100050 & $2^{-2}$ \\
$6^{t h}$ & 0x0100000000000500 & 0x0800000000000a00 & $2^{-4}$ \\
$7^{t h}$ & 0x0a00000000000800 & 0x0800000000000300 & $2^{-2}$ \\
$8^{t h}$ & 0x1a00000000000000 & 0x8400000000000000 & $2^{-4}$ \\
$9^{t h}$ & 0xc000000000000000 & 0x1000000000000000 & $2^{-1}$ \\
$10^{t h}$ & 0x0001000000000000 & 0x0008000000000000 & $2^{-2}$ \\
$11^{t h}$ & 0x0008000000000000 & 0x0004000000000000 & $2^{-2}$ \\
$12^{t h}$ & 0x0040000000000000 & 0x0060000000000000 & $2^{-1}$ \\
$13^{t h}$ & 0x0400002000000000 & 0x0600006000000000 & $2^{-2}$ \\
$14^{t h}$ & 0x4000060000200000 & 0x6000080000600000 & $2^{-3}$ \\
\hline
\end{tabular}

Table 14: The best differential characteristic with probability $2^{-72}$ for 16 -round LBLOCK.

\begin{tabular}{c|c|c|c}
\hline Rounds & input difference of S-boxes & output difference of S-boxes & probability \\
\hline $1^{t h}$ & 0x00424000 & 0x00218000 & $2^{-6}$ \\
$2^{t h}$ & 0x00040000 & 0x00020000 & $2^{-2}$ \\
$3^{t h}$ & 0x40400000 & 0x40200000 & $2^{-4}$ \\
$4^{t h}$ & 0x04420000 & 0x04650000 & $2^{-6}$ \\
$5^{t h}$ & 0x05060040 & 0x0000000000 & $2^{-6}$ \\
$6^{t h}$ & 0x00000000 & 0x0100c00a & $2^{0}$ \\
$7^{t h}$ & 0x06004005 & 0xf0000260 & $2^{-6}$ \\
$8^{t h}$ & 0x10000ac0 & 0x00a01c00 & $2^{-8}$ \\
$9^{t h}$ & 0x00b02500 & 0x00000000 & $2^{-6}$ \\
$10^{t h}$ & 0x000000000 & 0x20120000 & $2^{-6}$ \\
$11^{t h}$ & 0xb0250000 & 0x02150000 & $2^{-6}$ \\
$12^{t h}$ & 0x02210000 & 0xa000000000 & $2^{-4}$ \\
$13^{t h}$ & 0x000100b0 & 0x0a102000 & $2^{-2}$ \\
$14^{t h}$ & 0x20000000 & 0xe0050000 & $2^{-6}$ \\
$15^{t h}$ & 0x01a0b000 & & $2^{-4}$ \\
$16^{t h}$ & 0xa0010000 &
\end{tabular}

Table 15: The best linear characteristic with correlation $2^{-33}$ for 15 -round LBLOCK.

\begin{tabular}{c|c|c|c}
\hline Rounds & input mask of S-boxes & output mask of S-boxes & correlation \\
\hline $1^{t h}$ & 0x00000000 & 0x00000000 & $2^{0}$ \\
$2^{t h}$ & 0xd09d0000 & 0x10590000 & $2^{-3}$ \\
$3^{\text {th }}$ & 0x08053000 & 0x090dd000 & $2^{-3}$ \\
$4^{\text {th }}$ & 0x9d0000d0 & 0x31000010 & $2^{-3}$ \\
$5^{\text {th }}$ & 0x00000000 & 0x00000000 & $2^{0}$ \\
$6^{\text {th }}$ & 0x02007007 & 0x03001001 & $2^{-3}$ \\
$7^{\text {th }}$ & 0x30000033 & 0x70000027 & $2^{-3}$ \\
$8^{t h}$ & 0x00700702 & 0x00100203 & $2^{-3}$ \\
$9^{t h}$ & 0x00000000 & 0x00000000 & $2^{0}$ \\
$10^{t h}$ & 0xf07b0000 & 0x20310000 & $2^{-3}$ \\
$11^{t h}$ & 0x03032000 & 0x070bf000 & $2^{-3}$ \\
$12^{t h}$ & 0x7b0000f0 & 0x000000000 & $2^{-3}$ \\
$13^{t h}$ & 0x00000000 & 0x02002002 & $2^{0}$ \\
$14^{t h}$ & 0x0f001009 & 0x100000f9 & $2^{-3}$ \\
$15^{t h}$ & 0xe00000c1 & &
\end{tabular}


Table 16: The best differential characteristic with probability $2^{-68}$ for 15 -round TWINE.

\begin{tabular}{c|c|c|c}
\hline Rounds & input difference of S-boxes & output difference of S-boxes & probability \\
\hline $1^{t h}$ & 0x00000000 & 0x00000000 & $2^{0}$ \\
$2^{t h}$ & 0xca0c0000 & 0xe7020000 & $2^{-7}$ \\
$3^{\text {th }}$ & 0xe0702000 & 0xa090a000 & $2^{-7}$ \\
$4^{t h}$ & 0x00ca0050 & 0x00e70020 & $2^{-6}$ \\
$5^{t h}$ & 0x00000000 & 0x00000000 & $2^{0}$ \\
$6^{t h}$ & 0x00a0c005 & 0x000020009a & $2^{-7}$ \\
$7^{t h}$ & 0x00020072 & 0x02000207 & $2^{-6}$ \\
$8^{t h}$ & 0x0500050a & 0x00000000 & $2^{-6}$ \\
$9^{t h}$ & 0x00000000 & 0x27020000 & $2^{0}$ \\
$10^{t h}$ & 0x5a050000 & 0xa050a000 & $2^{-7}$ \\
$11^{t h}$ & 0x20702000 & 0x00270000 & $2^{-4}$ \\
$13^{t h}$ & 0x005a0000 & 0x00000a00 & $2^{-2}$ \\
$14^{t h}$ & 0x00000200 & 0x07702000 & $2^{-6}$ \\
$15^{t h}$ & 0x0aa05000 & 0x00900090 & $2^{-4}$ \\
$16^{t h}$ & 0x00700070 & & \\
\hline
\end{tabular}

Table 17: The best linear characteristic with correlation $2^{-35}$ for 16 -round TWINE.

\begin{tabular}{c|c|c|c}
\hline Rounds & input mask of S-boxes & output mask of S-boxes & correlation \\
\hline $1^{t h}$ & 0x00000000 & 0x00000000 & $2^{0}$ \\
$2^{t h}$ & 0xc60c0000 & 0x12010000 & $2^{-3}$ \\
$3^{\text {th }}$ & 0xb2200000 & 0x6cc00000 & $2^{-3}$ \\
$4^{t h}$ & 0x60c000c0 & 0x20a00010 & $2^{-3}$ \\
$5^{t h}$ & 0x00000000 & 0x00000000 & $2^{0}$ \\
$6^{t h}$ & 0x0060c00c & 0x0020100a & $2^{-3}$ \\
$7^{t h}$ & 0x000b0220 & 0x00060cc0 & $2^{-3}$ \\
$8^{t h}$ & 0x0c006c00 & 0x0a002a00 & $2^{-3}$ \\
$9^{t h}$ & 0x00000000 & 0x00000000 & $2^{0}$ \\
$10^{t h}$ & 0xce010000 & 0xa20a0000 & $2^{-3}$ \\
$11^{t h}$ & 0xad200000 & 0xe1c00000 & $2^{-3}$ \\
$12^{t h}$ & 0xe00000c0 & 0xd00000a0 & $2^{-2}$ \\
$13^{t h}$ & 0x000c0000 & 0x00010000 & $2^{-1}$ \\
$14^{t h}$ & 0x02e01000 & 0x0cd0a000 & $2^{-3}$ \\
$15^{t h}$ & 0xe00a0000 & 0x200e0000 & $2^{-2}$ \\
$16^{t h}$ & 0x00a2000e & 0x00ec000d & $2^{-3}$ \\
\hline
\end{tabular}

